\title{
1 Tree phyllospheres are a habitat for diverse populations of CO-oxidising bacteria
} 2

3 Jess L. Palmer*, Sally Hilton, Emma Picot, Gary D. Bending, Hendrik Schäfer*

4 School of Life Sciences, University of Warwick, Coventry, CV4 7AL, United Kingdom

5

6

$7 \quad *$ For correspondence:

8 Email: Jess.L.Palmer@warwick.ac.uk; H.Schaefer@warwick.ac.uk 


\section{Abstract}

Background: Carbon monoxide ( $\mathrm{CO}$ ) is a naturally occurring and ubiquitous trace gas in the atmosphere. As a product of combustion processes, it can reach concentrations in the $\mathrm{mg} / \mathrm{m}^{3}$ range in urban areas, contributing to air pollution. Aerobic CO-degrading microorganisms have been identified previously and are thought to remove $\sim 370 \mathrm{Tg}$ of $\mathrm{CO}$ in soils and oceans per year. Based on the presence of genes encoding subunits of the enzyme carbon monoxide dehydrogenase in metagenomes, a large fraction of soil bacteria may have the potential for $\mathrm{CO}$ degradation. The activity and diversity of CO-degrading microorganisms in above ground habitats such as the phyllosphere has not been addressed, however, and their potential role in global CO cycling remains unknown.

Results: Monitoring of CO-degradation in leaf washes of two common British trees, Ilex aquifolium and Crataegus monogyna, demonstrated CO uptake in all samples investigated. Leaf washes of $I$. aquifolium had significantly higher $\mathrm{CO}$ oxidation rates than those of $C$. monogyna. A diverse range of bacterial taxa were identified as candidate CO-oxidising taxa based on high-throughput sequencing and multivariate statistical analysis of $16 \mathrm{~S}$ rRNA amplicon data, as well as functional diversity analysis based on coxL, the gene encoding the large subunit of CO-dehydrogenase. Candidate CO-oxidising taxa included a range of Rhizobiales and Burkholderiales, of which the Burkholderiales OTUs were abundant colonisers of the phyllosphere at the time of sampling, as indicated by $16 \mathrm{~S}$ rRNA gene sequencing. In addition, an estimated $12.4 \%$ of leaf OTUs in samples of this study contained coxL homologues, based on their predicted genomes. We also mined data of publicly available phyllosphere metagenomes for genes encoding subunits of CO-dehydrogenase which indicated that, on average, $25 \%$ of phyllosphere bacteria contained CO-dehydrogenase gene homologues. A CO-oxidising Phyllobacteriaceae strain was isolated from phyllosphere samples which contains genes encoding both CODH as well as a RuBisCO.

Conclusions: The phyllosphere, a vast microbial habitat, supports diverse and potentially abundant CO-oxidising bacteria. These findings identify tree phyllosphere bacteria as a potential sink for atmospheric $\mathrm{CO}$ and highlight the need for a more detailed assessment of phyllosphere microbial communities in the global cycle of CO. 


\section{Introduction}

The phyllosphere, defined as the above ground parts of plants, is a vast microbial habitat covering an estimated surface area of around 1 billion $\mathrm{km}^{2}[4,5]$. It is colonised by diverse microorganisms including fungi, archaea, protists, viruses and bacteria, of which bacteria are the most abundant group, with an estimated $10^{6}-10^{7}$ bacterial cells per $\mathrm{cm}^{2}$ of leaf [6]. Phyllosphere microbial communities have taxonomically diverse bacterial communities that vary seasonally, geographically and with plant host [4, 7-10].

UV radiation, fluctuations in water availability and temperature, and scarcity of nutrients, characterise the phyllosphere as a harsh environment. Phyllosphere bacteria have been implicated in plant health, and interactions with the plant host range from symbiotic to pathogenic $[11,12]$. Phyllosphere bacteria also contribute to biogeochemical cycling and degradation of pollutants, as demonstrated by the degradation of plant or atmospherically derived organic compounds such as methanol [13], phenol [14], 4-chlorophenol [15], benzene [16], polycyclic aromatic hydrocarbons (PAHs) [17], methyl chloride [18] isoprene [19, 20] and diesel [21]. In doing so, activities of phyllosphere bacteria affect atmospheric chemistry and the fate of atmospheric pollutants and may thus contribute to critical ecosystem services of the phyllosphere in mitigation of air pollution, a major global public health problem causing around 5.5 million premature deaths worldwide [22].

Carbon monoxide (CO) is a ubiquitous component of the atmosphere. It is toxic to humans and even small changes in $\mathrm{CO}$ concentration in urban areas can have an impact on human health, with a $1 \mathrm{mg} / \mathrm{m}^{3}$ increase in CO being associated with a $4.4 \%$ increase in cardiovascular disease hospital admissions [23]. Atmospheric $\mathrm{CO}$ also negatively impacts the environment by reducing concentrations of hydroxyl radicals and thus increasing the residence times of greenhouse gases such as methane [24], contributing to the increase in ground-level ozone [25] and leading to the formation of photochemical smog [26].

Environmental sources of atmospheric CO include volcanic eruptions, bushfires, photochemical reactions and photolysis of marine coloured dissolved organic matter $(\mathrm{CDOM})[27,28]$. In addition, both live and dead plant matter have been shown to emit CO due to photochemical transformations within living leaf tissue or by UV-induced photoproduction by dead leaf tissue. This results in an estimated 50-200 $\mathrm{Tg} \mathrm{yr}^{-1}$ of CO and 
$60-90 \mathrm{Tg} \mathrm{yr}^{-1}$ of $\mathrm{CO}$ being produced from live plant tissues and dead plant matter, respectively $[29,30]$.

The major global source of $\mathrm{CO}$, however, is anthropogenic combustion processes which contribute more than half of annual CO emissions (approx. >1,200 Tg per year) [31, 32]. CO concentrations are highest in urban areas, ranging from $1.1-2.5 \mathrm{mg} / \mathrm{m}^{3}$ in the UK [33], compared to the atmospheric background of roughly $50-150 \mathrm{ppb}\left(0.058-0.17 \mathrm{mg} / \mathrm{m}^{3}\right)[34-$ 36]. However, in polluted cities such as Beijing, CO concentrations as high as $17.1 \mathrm{mg} / \mathrm{m}^{3}$ have been reported [37].

Most $\mathrm{CO}$ in the atmosphere is converted to $\mathrm{CO}_{2}$ via tropospheric hydroxylation [38], but significant amounts of $\mathrm{CO}$ are removed by microbial oxidation. Approximately $10-15 \%$ of the global CO flux is consumed by soil microbiota [39] which remove approximately $300 \mathrm{Tg}$ of CO per year [32]; marine microbiota are estimated to oxidise approximately $70 \mathrm{Tg}$ of CO per year [40], preventing the release of $85 \%$ of CO produced in the oceans into the atmosphere $[41,42]$. Despite the fact that a significant amount $\left(110-290 \mathrm{Tg} \mathrm{yr}^{-1}\right)$ of CO is produced by living and dead plant matter, it is currently unknown whether plant-associated microorganisms play a role in the mitigation of $\mathrm{CO}$ from this source.

Bacteria using $\mathrm{CO}$ as a carbon and energy source, so-called carboxydotrophs, typically utilise high concentrations of $\mathrm{CO}(>1 \%)$, assimilating the $\mathrm{CO}_{2}$ produced via the Calvin cycle. However, carboxydotrophs have a low affinity for CO, preferring other organic substrates [32]. Other CO-oxidisers, known as carboxydovores, cannot grow at elevated CO concentrations but oxidise CO up to $1000 \mathrm{ppm}$. Carboxydovores have a high affinity for CO but cannot generate biomass when $\mathrm{CO}$ is the sole carbon source; they likely use $\mathrm{CO}$ as a supplementary energy source [32].

Aerobic $\mathrm{CO}$-oxidising bacteria oxidise $\mathrm{CO}$ to $\mathrm{CO}_{2}$ using the enzyme carbon monoxide dehydrogenase $(\mathrm{CODH})$ of which two forms, referred to as form I and form II, have been described. Both enzymes are composed of three subunits, encoded by coxL (large subunit), coxM (medium subunit), and coxS (small subunit) [43]. The form I cox operon is transcribed in the order $M, S, L$ whereas the form II operon is transcribed in the order $S, L, M$. Form I CODH has been described as the 'definitive' form and has been well-characterised in several carboxydotrophs [44]. A form II enzyme was shown to be a functional CODH in Bradyrhizobium japonicum USDA 110 [45] and Kyrpidia spormannii [46], but the inability to oxidise $\mathrm{CO}$ by a range of bacteria harbouring exclusively form II CODH [47] suggests that some 
102

103

104

105

106

107

108

109

110

111

112

113

114

115

116

117

118

119

120

121

122

123

124

125

126

127

128

129

130

131

aspects of form II CODH function or regulation remain unknown. Carboxydotrophs and carboxydovores are taxonomically diverse, including members of the Proteobacteria, Firmicutes, Actinobacteria, Chloroflexi and Bacteroidetes identified in marine [47-49], soil $[48,50,51]$ and rhizosphere environments $[45,48]$. The presence of $\operatorname{cox} L$ genes in a wide range of environments and in representatives of uncultivated clades of bacteria indicates that the diversity of CO-oxidising bacteria is greater than those currently identified by cultivation $[49,52-58]$. CO oxidation is known to enhance the long-term survival of some bacteria, supporting their persistence in deprived or changeable environments [59] and recent research indicates that $\mathrm{CO}$ oxidation may be a more generalist function than previously assumed, with $56 \%$ of soil bacteria predicted to be capable of CO oxidation [60]. It is therefore possible that CO oxidation may be a widespread function in a range of microbial environments.

$\mathrm{CO}$ emissions from plant matter and anthropogenic sources such as vehicle exhaust fumes likely provides a constant input of $\mathrm{CO}$ to the leaf environment, suggesting that $\mathrm{CO}$ could be a nutrient exploited by bacteria in the nutrient-limited habitat of the leaf surface. Here we assessed the potential of phyllosphere microbial communities to degrade $\mathrm{CO}$ and identified CO-degrading microorganisms using a combination of cultivation-dependent and cultivationindependent approaches. We show that tree phyllosphere samples are capable of CO oxidation and that some samples oxidised $\mathrm{CO}$ even when no other organic carbon sources were provided. The phyllosphere communities of Ilex aquifolium (holly) and Crataegus monogyna (hawthorn) contained diverse populations of $\mathrm{CO}$ degrading bacteria based on highresolution microbial community analysis coupled to multivariate statistical analyses and functional marker diversity of coxL. A Phyllobacteriaceae strain able to oxidise CO was also isolated from phyllosphere samples. Overall, these results identify tree phyllospheres as a habitat for CO-oxidising bacteria and suggest that phyllosphere microorganisms contribute to global cycling of CO.

\section{Materials and Methods}

\section{Sample collection and processing}


132 Two species of common trees in Britain, hawthorn (Crataegus monogyna) and holly (Ilex

133 aquifolium) were investigated, providing samples from both a deciduous and evergreen tree

134 species. Trees were sampled on 6/10/2017. Tree branches were sampled in Coventry, United 135 Kingdom, with hawthorn trees sampled at both a woodland (sample code ' $\mathrm{HtW}$ ') (52.376248, $136-1.551322)$ and roadside (sample code 'HtR')(52.394371, -1.554182) location whereas holly 137 trees were sampled at the woodland site (sample code 'HIW') $(52.376248,-1.556771)$ only. 138 At each site, four trees were selected and whole twigs were removed at random from 1.5-2m 139 high and were placed into sterile, polyethylene zip-lock bags to be transported to the lab where they were processed within 24 hours.

Phyllosphere communities were collected by adapting a leaf washing method by Atamna-Ismaeel et al., [61]. In summary, $5 \mathrm{~g}$ of leaves per sample were weighed into sterile $250 \mathrm{ml}$ Erlenmeyer flasks, to which $150 \mathrm{ml}$ sterile phosphate-buffered saline (PBS) buffer (137 $\mathrm{mM} \mathrm{NaCl}, 2.7 \mathrm{mM} \mathrm{KCl}, 10 \mathrm{mM} \mathrm{Na}_{2} \mathrm{HPO}_{4}, 1.8 \mathrm{mM} \mathrm{KH}_{2} \mathrm{PO}_{4}, \mathrm{pH}$ 7.4) was added. Microorganisms and other particulates were then dislodged from the leaf surface by sonication for 2 minutes in a water bath, followed by 10 seconds of vortexing and a 5-minute resting period. The vortex and resting stages were repeated 6 times to thoroughly dislodge leaf surface material into the buffer. Leaf wash was filtered through $0.22 \mu \mathrm{m}$ pore size membrane filters (Millipore, USA). Leaf wash filters were cut in half and either immediately used to determine their potential for $\mathrm{CO}$ oxidation or immediately frozen and stored at $-80^{\circ} \mathrm{C}$ for future DNA extraction.

\section{Assessment of leaf wash CO oxidation potential and cultivation of phyllosphere microbial} communities enriched with CO

Sterile mineral salts medium was prepared for use in $\mathrm{CO}$ degradation experiments and enrichment of CO-degrading microorganisms as described by Meyer and Schlegel [44]. Analytical grade chemicals were dissolved in Milli-Q water before sterilisation by autoclaving at $121^{\circ} \mathrm{C}$ for 15 minutes. To encourage the heterotrophic oxidation of $\mathrm{CO}, 0.25 \mathrm{~g} / \mathrm{L}$ of yeast extract and $1 \mathrm{~mL}$ of filter sterilised vitamin solution [62] was added to the mineral salts medium. Solid media for isolation of CO-oxidising bacteria were prepared accordingly with addition of 1.5\% (w/v) BactoAgar (Becton Dickinson, USA). Liquid media ( $25 \mathrm{~mL}$ ) added to 125 $\mathrm{mL}$ serum vials were inoculated by adding half of a leaf wash filter per vial. Vials were sealed with a butyl plug, crimp-sealed, and CO was injected into the headspace to a concentration 
of approximately $800 \mathrm{ppm}$. Sterile controls were prepared without inoculum and nosubstrate controls were prepared containing the microbial inoculum but without addition of CO. Enrichment cultures and controls were incubated in the dark in a shaking incubator at $22^{\circ} \mathrm{C}$ and $100 \mathrm{rpm}$.

Headspace CO concentrations were monitored every 2-4 days using an Agilent 6890N gas chromatograph (GC; Agilent Technologies, California, US) equipped with a nickel catalyst methaniser (Agilent Technologies, California, US) allowing detection of $\mathrm{CO}$ after its reduction to methane using a flame ionisation detector. The system was fitted with a $1.5 \mathrm{~m}$ long 80/100 Porapak Q column (inner diameter $2.1 \mathrm{~mm}$; Sigma-Aldrich, US) held at $250^{\circ} \mathrm{C}$ using nitrogen $(50 \mathrm{~mL} / \mathrm{min})$ used as the carrier gas. A $100 \mu \mathrm{l}$ headspace sample was injected into the apparatus and peak readings for CO were taken. $\mathrm{CO}$ headspace concentrations in the headspace of enrichment cultures were calculated based on a calibration derived from injection of samples of known CO concentrations. $\mathrm{CO}$ concentrations between 0 (ambient air) and $50 \mathrm{ppm}$ could not be differentiated and so $<50 \mathrm{ppm}$ was defined as the detectable limit. prepared for both the enrichment sample and its corresponding control sample. Enrichment sub-cultures were prepared and monitored as above, using $2 \mathrm{~mL}$ of liquid culture from the corresponding sample as the microbial inoculum added to $23 \mathrm{~mL}$ of fresh mineral salts medium (plus yeast extract). This was repeated twice upon the degradation of $\mathrm{CO}$ for a total of three sub-culture cycles per sample. Once the final sub-culture had degraded the $\mathrm{CO}, 15$ $\mathrm{mL}$ of liquid culture was extracted and centrifuged at $4500 \times \mathrm{g}$ for 20 minutes to form a pellet. The supernatant was removed and the pellet was flash frozen in liquid nitrogen before storing at $-80^{\circ} \mathrm{C}$ for future DNA extraction.

Once individual samples had degraded CO on their final sub-culture, they and their corresponding no-substrate control culture were also subcultured into mineral salts media without addition of yeast extract so that $\mathrm{CO}$ was the sole carbon source in order to investigate the occurrence of autotrophic degradation of CO. These 'autotrophic' subcultures were prepared by centrifugation of $15 \mathrm{~mL}$ of the final 'heterotrophic' sub-culture at $4500 \times \mathrm{g}$ for 10 minutes, discarding the supernatant and resuspending the pellet in $2 \mathrm{~mL}$ of autotrophic CO. This was repeated three times before the washed pellet resuspension was added as an inoculum to $23 \mathrm{~mL}$ of mineral salts media without yeast extract. Degradation of CO was monitored and cultures were re-spiked with $\mathrm{CO}$ to bring the headspace concentration back 
to $800 \mathrm{ppm}$ upon depletion. A total of three re-spikes were monitored and upon degradation of the final $\mathrm{CO}$ pulse, $15 \mathrm{~mL}$ of liquid culture was processed as described for heterotrophic CO enrichment cultures above for isolation of colonies and DNA extraction.

\section{DNA extraction}

DNA from CO enrichment cultures and no-substrate controls was extracted using the FastDNA $^{\mathrm{TM}}$ Spin Kit for Soil (MP Bio Science Ltd., Derby, UK). The required amount of sodium phosphate buffer and MT buffer was added to the enrichment culture pellet in order to resuspend the pellet before being transferred to the Lysing Matrix E tube and proceeding with the protocol as per the manufacturer's instructions. At the final step, DNA was eluted using $50 \mu \mathrm{L}$ of elution buffer. Aliquots of DNA extracts were assessed for quantity and quality by gel electrophoresis using $0.8 \%(\mathrm{w} / \mathrm{v})$ agarose gels and a Qubit ${ }^{\circledR} 2.0$ Fluorometer (Invitrogen, USA), using high-sensitivity reagents.

\section{S rRNA PCR and MiSeq library preparation}

High-throughput sequencing was used to investigate the diversity of $16 \mathrm{~S}$ rRNA genes in CO enrichment culture samples, corresponding no-substrate controls and original leaf wash samples. For each sample, $20 \mathrm{ng}$ of DNA was used as template to amplify $292 \mathrm{bp}$ of $16 \mathrm{~S}$ rRNA genes using the $515 f\left(5^{\prime}\right.$-GTGCCAGCMGCCGCGGTAA-3') and the $806 \mathrm{r}$ (5'GGACTACHVGGGTATCTAAT-3') primer pair [63] with Illumina Nextera Transposase Adapters attached to the $5^{\prime}$ end. PCRs were performed in $25 \mu \mathrm{L}$ reaction volumes and contained $13 \mu \mathrm{L}$ Q $5^{\circledR}$ Hot Start High-Fidelity $2 \times$ Master Mix (New England Biolabs, US), $0.5 \mu \mathrm{M}$ of each primer and $0.4 \mu \mathrm{L}$ of $25 \mathrm{mg} / \mathrm{mL}$ BSA. The reaction conditions were as follows: an initial denaturing step of $98^{\circ} \mathrm{C}$ for $30 \mathrm{~s}$ followed by 25 cycles of $98^{\circ} \mathrm{C}$ for $10 \mathrm{~s}, 50^{\circ} \mathrm{C}$ for $15 \mathrm{~s}$ and $72^{\circ} \mathrm{C}$ for $20 \mathrm{~s}$ and a final extension of $72^{\circ} \mathrm{C}$ for 10 mins. PCR products were then purified using the AmpliClean ${ }^{\mathrm{TM}}$ Magnetic Bead PCR Clean-Up Kit (NIMAGEN, Nijmegen, Netherlands), which was carried out as per the manufacturer's instructions.

Purified PCR products were given unique dual indexes at the $5^{\prime}$ end using the Nextera XT Index Kit v2 index primers (Illumina, USA). To attach the index primers, a PCR mixture was made consisting of $13 \mu \mathrm{L} \mathrm{Q5}{ }^{\circledR}$ Hot Start High-Fidelity $2 \times$ Master Mix, $1 \mu \mathrm{M}$ Index Primer 1, 1 $\mu \mathrm{M}$ Index Primer 2 and $4 \mu \mathrm{l}$ of purified PCR product, with the addition of sterile, nuclease-free 
$\mathrm{dH}_{2} \mathrm{O}$ for a final reaction volume of $26 \mu \mathrm{L}$ per sample. The unique indexes were added by amplifying PCR products with the following reaction conditions: $95^{\circ} \mathrm{C}$ for 3 mins, followed by $8 \mathrm{cycles}$ of $98^{\circ} \mathrm{C}$ for $20 \mathrm{~s}, 55^{\circ} \mathrm{C}$ for $15 \mathrm{~s}$, and $72^{\circ} \mathrm{C}$ for $15 \mathrm{~s}$, then a final elongation step of $72^{\circ} \mathrm{C}$ for 5 mins. Samples were normalised using the SequalPrep ${ }^{\mathrm{TM}}$ Normalisation Plate Kit (Invitrogen, USA) as per the manufacturer's instructions. All samples were pooled and adjusted to $4 \mathrm{nM}$ before being submitted to the Genomics Facility at the University of Warwick, Coventry, United Kingdom for sequencing on the Illumina Miseq platform.

\section{coxL functional gene PCR amplification and construction of clone libraries}

Amplification of form I coxL genes using DNA from CO enrichment cultures was initially attempted using the OMPf and $\mathrm{O} / \mathrm{Br}$ coxL primer pair [48]. As this was not successful, this primer pair was reviewed and modified. Form I coxL sequences were obtained from the Integrated Microbial Genome and Metagenome (IMG) database [64] using the BLASTx function with a Burkholderia fungorum form I coxL sequence (AY307914.1) as a query. Fivehundred extra form I coxL genes were obtained in addition to the nine form I coxL genes used for the design of the original OMPf O/Br primer pair which were then aligned using ARB software [65] and a neighbour-joining tree was constructed. Primer sequences were reviewed with focus on coxL genes from terrestrial origins. An alignment was made with a total of 437 coxL sequences, the diversity of which is displayed in Figure S1. Mismatches were identified and modifications made to increase the redundancy of the primers in an attempt to improve upon the range of bacterial species targeted by the primers. Modifications made are shown in Table S1.

The newly modified forward (5'- GGCGGNTTYGGNAAYAARGT) and reverse (5'YTCDATDATCATNGGRTTDAT) primer pair was used to amplify definitive (form I) coxL genes from samples. PCRs were prepared containing 1x KAPA Taq Buffer A (includes $1.5 \mathrm{mM} \mathrm{MgCl}_{2}$ ) (Kapa Biosystems, USA), dNTP nucleotide mix (0.2 mM each), DMSO (2\% v/v), BSA $(0.05 \%$ $w / v)$, forward and reverse primer $(0.4 \mu \mathrm{M}$ each), KAPA Taq DNA polymerase $(2 \mathrm{U})$ (KAPABIOSYSTEMS) and DNA template $(2-20 \mathrm{ng})$. The reaction was made up to a $50 \mu \mathrm{L}$ reaction volume with sterile nuclease-free $\mathrm{dH}_{2} \mathrm{O}$. Positive controls were included which contained DNA template from Ruegeria pomeroyi DSS-3, a known CO oxidiser. Negative controls did not contain any DNA template. Reaction conditions were as follows: initial denaturation at $94^{\circ} \mathrm{C}$ for $5 \mathrm{~min}$, followed by 35 cycles of a $30 \mathrm{~s}$ denaturation at $94^{\circ} \mathrm{C}, 30 \mathrm{~s}$ 
annealing at $56^{\circ} \mathrm{C}$ and $1: 15$ min extension at $72^{\circ} \mathrm{C}$, with a final extension of 7 min at $72^{\circ} \mathrm{C}$. PCR products were visualised with GelRed (Biotium, USA) gel electrophoresis and gel-purified. PCR products were sequenced by Sanger sequencing either by GATC Biotech GmbH (Konstanz, Germany) or Eurofins Genomics (Ebersberg, Germany).

The diversity of coxL genes in $\mathrm{CO}$ enrichment culture samples was assessed by cloning purified PCR products using the TOPO TA cloning kit and TOP10 competent cells (Invitrogen, USA) as per the manufacturer's instructions. Transformants were used as template for PCR amplification of coxL using the modified primer pair. Products were purified and sequenced as described previously.

\section{Isolation, identification and genome sequencing of CO-oxidising bacteria}

Microbial culture $(100 \mu \mathrm{l})$ sampled from heterotrophic or autotrophic enrichment vials after the final sub-culture was serially diluted with mineral salts media [44]. Dilutions were spread plated onto mineral salts plates (containing $0.25 \mathrm{~g} / \mathrm{L}$ yeast extract) and LB plates and incubated at $22^{\circ} \mathrm{C}$ in the dark. Single colonies were selected at random and screened by PCR for presence of coxL gene as described previously. If products of the correct size were amplified, the corresponding colony was then re-suspended into mineral salts medium with 800 ppm CO and monitored for CO degradation by GC.

For taxonomic identification of isolates, the 16S rRNA gene was amplified as described above for coxL PCR but using the $27 f$ (5'- AGAGTTTGATCMTGGCTCAG) and 1492r (5'TACGGYTACCTTGTTACGACTT) primer pair [66] and using $1 \mu \mathrm{l}$ of cell suspension from isolates that had first been heated at $100^{\circ} \mathrm{C}$ for $5 \mathrm{~min}$. Reaction conditions were as follows: initial denaturation at $94^{\circ} \mathrm{C}$ for $5 \mathrm{~min}$, followed by 35 cycles of a $30 \mathrm{~s}$ denaturation at $94^{\circ} \mathrm{C}, 30 \mathrm{~s}$ annealing at $55.5^{\circ} \mathrm{C}$ and $1: 15$ min extension at $72^{\circ} \mathrm{C}$, with a final extension of 7 min at $72^{\circ} \mathrm{C}$. PCR products were visualised, purified and sequenced as described previously.

For whole genome sequencing of the CO-oxidising isolate, a lawn of cells was grown on an LB plate before harvesting of the cells which were sent to MicrobesNG (Birmingham, UK) where DNA was extracted and sequenced using the Illumina HiSeq platform (250bp paired-end protocol). Adapters were trimmed and assembled by MicrobesNG using Trimmomatic [67] and SPAdes version 3.7 [68], respectively. Contigs were then submitted to RAST [69] for annotation and analysis of CO oxidation gene clusters. 
291

292

293

294

295

296

297

298

299

300

301

302

303

304

305

306

307

308

309

310

311

312

313

314

315

316

317

318

319

320

321

\section{Bioinformatic and statistical analyses}

For the processing of MiSeq data, low-quality sequences were removed from the ends of the pre-demultiplexed reads using Trimmomatic version 0.36 [67]. USEARCH [70] was then used for the majority of downstream analyses. Paired ends were joined, quality filtering was applied to a $280 \mathrm{bp}$ minimum, primers were removed, and samples were dereplicated to remove any singletons. OTUs were clustered to a $97 \%$ identity threshold which also removed the majority of chimeras. Remaining chimeras were removed using the GOLD database [71] for $16 \mathrm{~S}$ rRNA amplicons. Reads were then mapped back to the OTU database for generation of OTU tables.

QIIME [72] was used along with the Greengenes [73] reference database to assign taxonomy to $16 \mathrm{~S}$ rRNA gene amplicons. OTU tables were generated and any OTUs identified as mitochondria or chloroplasts were removed. Samples were rarefied to 9000 reads per sample defined by the sample with the lowest number of reads.

Non-metric multi-dimensional scaling (MDS) analysis of Bray Curtis similarity of bacterial community structure were conducted using the VEGAN package in $\mathrm{R}$ [74]. Analysis of Similarity (ANOSIM) tests were used to determine how dissimilar bacterial communities were between groups using PRIMER v6 software [75]. Candidate CO-oxidising OTUs were identified by a combination of Similarity Percentages (SIMPER) analysis using PRIMER v6 software [75], to find those which drove the most dissimilarity between CO enrichment cultures and controls, and identification of OTUs which showed a significant log2fold increase in CO enrichment cultures compared to controls, which was done using the DESeq2 package [76] and visualised using the EnhancedVolcano package [77] in RStudio.

Significant differences in CO degradation times between sample types were calculated using Wilcoxon tests in R studio (version 1.1.447, RStudio Inc., US) to calculate P values.

All sequence traces were checked for quality using 4Peaks (Nucleobytes.com, Aalsmeer, The Netherlands) and high-quality sequence was used as a query for BLASTn (nucleotide BLAST for 16S rRNA sequences) or BLASTx (translated nucleotides protein BLAST for functional genes) searches [78]. Sequences were aligned with their top hits from the BLAST database using MUSCLE [79] in MEGA7 [2]. Phylogenetic trees were constructed using the neighbour-joining algorithm [1] with a bootstrap test applied [3] with 500 replicates in MEGA7. 
323 leaf wash community to oxidise CO, based on 16S rRNA amplicon data. 10649 OTUs were used in the analysis, of which 9701 (91\%) had a closely related genome available on the IMG database. From the predicted genomes of the OTUs and the relative abundance of each OTU (based on 16S rRNA data), the predicted copy number of coxL (KEGG K03520) was then compared between leaf wash sample types.

328

\section{Mining of CODH homologues from phyllosphere metagenomes}

330 In addition to predictions made by PICRUSt2 of the abundance of $\operatorname{cox} L$ genes from samples in this study, the metagenomics-rapid annotation using subsystems technology (MG-RAST) database [81] was used to determine how abundant $\mathrm{CODH}$ genes are in available phyllosphere metagenomes. Abundance data for protein homologues (identified by the MGRAST pipeline) of CODH subunits (CoxL, CoxM and CoxS), methanol dehydrogenase subunits ( $M x a F$ and Mxal) and three housekeeping genes (recA, rho and $r p o A$ ) were extracted from all publicly available phyllosphere metagenomes which included clover, soybean, Arabidopsis thaliana [82], rice [8] and 48 neotropical tree samples [83]. The abundances of Cox and methanol dehydrogenase homologues were then normalised against the three housekeeping genes to determine the relative abundance of the target functional genes in the bacterial communities. Attempts to align a sub-set (77 AA sequences) of clover metagenome CoxL amino acid sequences in order to confirm that sequences were CoxL homologues was unsuccessful due to the short length ( 60-90 AA) of partial sequences of CoxL homologues. However, BLASTp searches indicated that the majority had high (>70\%) AA sequence identity to other CoxL or xanthine dehydrogenase $(\mathrm{XDH})$ homologues. In addition, when the same subset (77 AA sequences) of CoxL homologues were aligned with both characterised CoxL [48] and xanthine dehydrogenase large subunits [84], the majority (68/77) had a higher sequence identity with CoxL than with xanthine dehydrogenase sequences.

\section{Results}

350 
352

353

354

355

356

357

358

359

360

361

362

363

364

365

366

367

368

369

370

371

372

373

374

375

376

377

378

379

380

381

382

383

Incubation of leaf wash samples from all three sample types, woodland hawthorn (HtW), roadside hawthorn ( $\mathrm{HtR}$ ) and woodland holly (HIW) in mineral medium amended with yeast extract resulted in the degradation of 800 ppm CO (Figure 1). On average, it took 33 days for $\mathrm{CO}$ to be degraded beyond the detectable limit $(<50 \mathrm{ppm})$ by all phyllosphere samples $(n=12)$, although there was variation in different replicate samples (range of 17-52 days). This demonstrated that leaf wash samples, and hence phyllosphere microbial communities, contained viable microorganisms capable of CO degradation.

The length of lag phases between samples also varied, with 5/8 hawthorn samples ( $\mathrm{HtW}$ and $\mathrm{HtR}$ ) showing long lag phases of between 17-30 days whereas some hawthorn samples (3/8) and all holly samples had short lag phases of 2-5 days before degradation of CO began. Once degradation of CO began, woodland samples degraded CO faster (average 45 ppm and 39 ppm CO per day for HtW and HIW samples, respectively), than the roadside hawthorn samples (24 ppm CO per day).

Woodland holly (HIW) samples degraded the CO the fastest (average 18.5 days), followed by woodland hawthorn ( $\mathrm{HtW}$ ) samples (average 37.25 days). Roadside hawthorn $(\mathrm{HtR})$ samples were the slowest (average of 43 days). There was no significant difference between $\mathrm{CO}$ degradation rates of $\mathrm{HtW}$ and $\mathrm{HtR}$ samples ( $\mathrm{P}$ value $=0.49$ ). However, $\mathrm{HIW}$ samples degraded CO significantly faster than $\mathrm{HtW}(\mathrm{P}$ value $=0.0037)$ and $\mathrm{HtR}(\mathrm{P}$ value $=$ 0.013) samples.

Once each sample had been sub-cultured three times upon CO degradation, the samples were inoculated into minimal media with $\mathrm{CO}$ but without additional carbon source to potentially indicate the presence of autotrophic CO oxidisers. Only half of all samples subcultured in this way continued to fully degrade three spikes of $800 \mathrm{ppm} \mathrm{CO}$, suggesting that some of the enrichments contained carboxydotrophic (autotrophic) CO oxidisers in addition to carboxydovores (Figure S2). It is noteworthy that those cultures which continued to degrade $\mathrm{CO}$ generally showed increasing rates of $\mathrm{CO}$ degradation which might indicate increased abundance of autotrophic $\mathrm{CO}$ oxidisers.

\section{Candidate $\mathrm{CO}$ oxidisers in the phyllosphere include rare and abundant community members}

Following complete $\mathrm{CO}$ degradation of three consecutive sub-cultures or re-spikes, the diversity of the enrichment cultures and no-substrate controls was assessed by highthroughput sequencing of $16 \mathrm{~S}$ rRNA genes. Analyses of the bacterial community composition 
outlined below revealed how leaf surface communities responded to co during the incubations and identified candidate $\mathrm{CO}$ oxidisers.

ANOSIM statistics showed that CO enrichment cultures were weakly but significantly dissimilar to their corresponding no-substrate controls, irrespective of whether they contained additional yeast extract as carbon source or not $(R=0.31, P=0.001$ and $R=0.24, P=$ 0.02 , respectively), as shown in Figure 2. Differences in bacterial community composition between $\mathrm{CO}$ enrichment cultures and controls at the OTU level are shown in Figures S3 and S4. No significant dissimilarities were found in the community composition of cultures inoculated with leaf wash from different tree species or tree locations (data not shown).

SIMPER analysis identified those OTUs that drove the dissimilarity between the leaf wash cultures enriched with $\mathrm{CO}$ and no-substrate controls, thus highlighting candidate $\mathrm{CO}$ oxidisers. For this purpose, we initially considered candidate CO oxidisers as those OTUs that were amongst the top ten OTUs driving dissimilarity between $\mathrm{CO}$ and no-substrate controls, and which showed $>1 \%$ increase in relative abundance in the $\mathrm{CO}$ enrichment cultures versus the no-substrate controls (Table 1). The majority of these OTUs were present at low relative abundances in the unenriched leaf wash samples. However, three candidate CO oxidiser OTUs (OTU9, OTU36 and OTU10) together comprised $>0.5-5.5 \%$ of total leaf wash community reads, on average. Strikingly, OTU10 (Comamonadaceae) had high relative read abundance (range 2.1-10.1\%) in all unenriched leaf wash communities suggesting it could be an abundant coloniser of the phyllosphere.

Using the Deseq2 package, we further considered as potential candidate CO oxidisers all of those OTUs which showed a log2-fold increase of $>1$ and a $P$ value of $<0.05$ in cultures enriched with CO versus no-substrate controls (Table 2 and Figure S5). Four of these OTUs (OTU792, OTU90, OTU33 and OTU5) had high read abundances (range 3.5 to $6.7 \%$ ) in the CO enrichment cultures and had also been identified by SIMPER analysis. Therefore, in addition to contributing to the most dissimilarity between $\mathrm{CO}$ enrichment culture and control groups, these OTUs also showed a significant log2-fold change. All other candidate CO oxidiser OTUs identified using this approach were not identified by SIMPER analysis and were present in CO enrichment cultures at low relative read abundances which were generally $<1 \%$ (except OTU87, 2.1\%). Similar to candidate CO-oxidiser OTUs identified by SIMPER analysis, most of the OTUs identified in Table 2 had low relative read abundance $(<0.1 \%)$ in the unenriched leaf 
and $0.37 \%$ of unenriched leaf wash reads on average, respectively. Additionally, OTU13,

417 which showed a significant log2-fold change in the 'autotrophic' CO enrichment culture was

418 abundant in the leaf wash samples, comprising $2 \%$ on average of reads of woodland hawthorn samples, $1.4 \%$ of roadside hawthorn samples and $4.3 \%$ of woodland holly samples. Taken together, these analyses suggest that candidate CO oxidisers in the phyllosphere microbiomes included both rare and abundant community members.

\section{Candidate $\mathrm{CO}$ oxidisers in the phyllosphere include diverse bacterial taxa}

424 The candidate CO oxidising OTUs identified above (Table 1 and 2) included a diverse range of bacteria belonging to five different phyla (Figure 3). The majority of candidate $\mathrm{CO}$ oxidisers were Proteobacteria (19/31), followed by Bacteroidetes (6/31), Actinobacteria (4/31), Deinococcus-Thermus (1/31) and Gemmatimonadetes (1/31). Three orders of bacteria contained the highest number of candidate CO-oxidising OTUs, four OTUs each were members of Burkholderiales, Xanthomonadales and Rhizobiales.

There were three groups of OTUs which clustered within their treatment group (CO plus yeast extract [Fig. 3, labelled in green] or CO only [Fig.3, labelled in blue]). The four Rhizobiales OTUs (OTU90, OTU269, OTU1705 and OTU72) were all enriched on CO when it was the sole carbon source, whereas the three Saprospirales (OTU279, OTU235 and OTU325) and the three Bacteroidetes (OTU67, OTU128, OTU4556) were identified in CO plus yeast extract incubations. Two OTUs were identified in both types of enrichments (Fig. 3, labelled purple). This analysis suggests that the phylogenetic diversity of candidate CO oxidising bacteria in the studied tree phyllospheres was high and that these might represent both carboxydotrophs and carboxydovores.

Analysis of coxL diversity within CO enrichment cultures was achieved by PCR amplification, cloning and sequencing of coxL using three CO-enriched samples (one per sample type) with added yeast extract. Ten transformant colonies containing an insert were used in subsequent coxL PCRs and sent for sanger sequencing, resulting in 28/30 high-quality sequences (Figure 4). 
being most closely related to coxL genes from a Rhizobiales bacterium and one sequence having the highest similarity to a Bradyrhizobium coxL gene. Four different clades of coxL originated from roadside hawthorn (HtR) samples, two of which were most similar to Rhizobiales, e.g. Rhizobium selenitireducens (2/9) and 'Rhizobiales bacterium 62-47' (5/9). A further two coxL types detected in the HtR sample were most closely related to $\operatorname{coxL}$ from Rhodococcus and Arthrobacter species, respectively. The majority of woodland holly (HIW) coxL sequences (5/10) were most similar to Burkholderia coxL genes. The remaining sequences were most similar to coxL from Afipia (2/10) or an Acidimicrobiia species (2/10). One of the sequences (HIW clone 10) had the highest similarity to a Xanthine dehydrogenase rather than a coxL gene. Thus, despite the limited depth of sequencing, the analysis of cloned coxL amplicons revealed a relatively high diversity of phyllosphere bacteria with the potential to degrade $\mathrm{CO}$ in the enrichment cultures. It is noteworthy that the closest relatives of $\operatorname{coxL}$ identified were in good agreement with some candidate CO oxidisers identified based on analysis of the $16 \mathrm{~S}$ rRNA sequencing data, i.e. members of the Rhizobiales, Burkholderiales, and Actinobacteria. None of the cloned coxL sequences were identical to the coxL gene of CO17, a CO-oxidising phyllosphere isolate (discussed below).

PICRUSt2 [85] was used to predict how many OTUs in the leaf wash samples had a close relative which had a coxL homologue, the gene encoding the large subunit of COdehydrogenase, in their genome sequence. 948 OTUs were removed from the sample group as they did not have a close relative in the database, leaving 9701 (91\%) OTUs in the analysis. Of these, PICRUSt2 analysis predicted that $12.4 \%$ (1202) of the leaf wash OTUs could have a $\operatorname{cox} L$ gene. In addition, the predicted copy number of $\operatorname{coxL}$ genes differed between some leaf wash sample types. On average, woodland holly samples contained the highest copy number of predicted coxL genes (5401) followed by woodland hawthorn (4867), with roadside hawthorn showing the lowest predicted coxL copy number (3755). While there was no significant difference $(P=<0.05)$ between the hawthorn (woodland and roadside) sample types, or the woodland sample types (hawthorn and holly), woodland holly samples had a significantly higher predicted coxL copy number than roadside hawthorn samples $(P=0.03)$.

Furthermore, the relative abundance of cox gene homologues (normalised with three single-copy housekeeping genes) within phyllosphere metagenomes available on the MGRAST database (clover, soybean, Arabidopsis thaliana, rice and neotropical trees) was determined using functional abundance data available on MG-RAST [81]. As displayed in 
480

481

482

483

484

485

486

487

488

489

490

491

492

493

494

495

496

497

498

499

500

501

502

503

504

505

506

507

508

509

510

Figure 5, the relative abundance of Cox gene homologues ranges between $21.7-55.3 \%$ for coxL, $10.4-27.3 \%$ for coxM and $9.3-28.1 \%$ for coxS genes. By comparison, the relative abundance of homologues of genes involved in methanol metabolism, $m x a F$ and $m x a l$ were between $0.5-25.9 \%$ and $0-9.8 \%$, respectively. Overall, the average relative abundance of CODH genes (coxL, coxM and coxS) (25\%) was higher than methanol dehydrogenase genes $(7.5 \%)$ in the phyllosphere metagenomes which were analysed.

\section{A Phyllobacteriaceae isolate, strain CO17, isolated from phyllosphere samples is capable of}

\section{CO oxidation}

Over 20 isolates were obtained from $\mathrm{CO}$ enrichment cultures and screened for their ability to degrade $\mathrm{CO}$, and the presence of a coxL gene. Only one of these isolates, designated CO17, was confirmed to oxidise CO. CO17 oxidised 800 ppm CO in the presence of yeast extract as an additional carbon source and while CO17 also oxidised $800 \mathrm{ppm}$ of CO without an additional carbon source, autotrophic growth on $\mathrm{CO}$ as a sole carbon source could not be confirmed. Sequencing of the 16S rRNA gene identified isolate CO17 as closely related to Mesorhizobium zhangyense, with a 99.7\% 16S rRNA sequence identity (Figure 6A). The 16S rRNA gene of isolate CO17 is distinct from that of OTU72 (a Mesorhizobium species identified in MiSeq analysis as a candidate CO oxidiser), sharing only $95 \%$ sequence identity. A draft genome sequence was obtained for isolate CO17 consisting of an assembly size of 7,199,351 bp (number of contigs 132, N50: 165,070; GC content 61.1\%). The genome assembly of strain CO17 has operons encoding both form I and form II CODH, the arrangements of which are typical of cox genes in other CO-oxidising bacterial species (Figure 6B). In addition, the genome of CO17 contained homologues of both the small ( $c b b S)$ and large ( $c b b L)$ RubisCO subunits. The $c b b L$ homologue of $\mathrm{CO} 17$ clustered within $c b b L$ genes which encode the definitive form I red-like RubisCO proteins, as shown in Figure S6. This indicates that CO17 may be able to assimilate of $\mathrm{CO}_{2}$, the oxidation product of $\mathrm{CO}$ in autotrophic $\mathrm{CO}$ oxidisers.

\section{Discussion}

Although CO oxidisers have been identified previously in a range of environments, their presence in the phyllosphere has only been speculative [32]. This work provides the first evidence that there are microorganisms in the phyllosphere which are capable of CO 
511 oxidation, with all leaf wash samples in this study oxidising $800 \mathrm{ppm}$ CO when cultivated with

512 an additional carbon source. Furthermore, there may be differences in the CO-oxidising

513 potential of different phyllosphere communities. Holly phyllosphere samples oxidised 800

514 ppm of CO significantly faster than hawthorn samples whereas there was no significant

515 difference between the time taken to oxidise CO between hawthorn tree samples taken from

516 the woodland versus the roadside. It is therefore possible that tree species has a greater

517 impact on the ability of phyllosphere communities to oxidise CO than location. This may be

518 due to the evergreen holly trees having a more established phyllosphere community than the

519 hawthorn trees at the time of sampling or it may be that differences in leaf physiology

520 between the tree species may form selective pressures on the phyllosphere community, thus

521 altering the community composition and functional capabilities of the phyllosphere. PICRUSt

522 data supported the idea that holly phyllosphere communities may have a higher capacity for

523 CO oxidation, with holly samples containing the highest predicted coxL gene abundance.

524 These initial data support further work that should include a wider range of tree species in

525 order to investigate potential correlations of tree species and CO-oxidising potential of the phyllosphere.

When $\mathrm{CO}$ enriched leaf wash cultures were sub-cultured into media where $\mathrm{CO}$ was a sole carbon source, only $50 \%$ of samples were able to consistently oxidise CO. This could indicate that microorganisms capable of autotrophic $\mathrm{CO}$ oxidation (carboxydotrophs) are not as abundant as heterotrophic CO oxidisers (carboxydovores). This is not surprising as atmospheric $\mathrm{CO}$ would be available to phyllosphere microorganisms at low concentrations ( 1-2 ppm in the UK) and therefore may better support microbial populations as a supplementary carbon source. Recent findings by Cordero et al. [59] showed that CODH expression is upregulated in response to starvation and, due to the abundance of CODH in soil and marine samples, they predicted that $\mathrm{CO}$ supported the persistence of aerobic heterotrophic bacteria in nutrient limited environments. Thus, results from this study indicating that heterotrophic $\mathrm{CO}$ oxidation may be more common than autotrophic $\mathrm{CO}$ oxidation in phyllosphere communities, the $\mathrm{CO}$ survival-based model of $\mathrm{CO}$ oxidation proposed by Cordero et al. [59] could also be an ecological adaptation enhancing survival in the phyllosphere, which is known to be deprived of energy sources. 
543 substrate controls may be due to toxic impacts of $\mathrm{CO}$ on the bacterial community, multiple

544 bacterial species were identified which were potentially enriched on CO. Three candidate CO-

545 oxidising OTUs where phylogenetically similar to genera which contain species proven to

546 oxidise CO [44, 45, 48, 86]. These are OTU9 (a Pseudomonas species), OTU72 (a

547 Mesorhizobium species) and OTU90 (similar to Bradyrhizobium species). The majority of

548 candidate $\mathrm{CO}$ oxidisers therefore present species for which $\mathrm{CO}$ oxidation would be a novel

549 observation for the genus they belong to. Notable examples include an Actinomycetospora

550 species (OTU13), a Phenylobacterium species (OTU33), a Janthinobacterium species (OTU20)

551 and a Luteimonas species (OTU5). All of which were significantly enriched in the presence of

$552 \mathrm{CO}$, indicating that $\mathrm{CO}$ oxidation is a likely function of these species. In addition, candidate

553 CO-oxidising OTUs were identified within classes of bacteria which are not yet documented

554 to contain CO-oxidising species. Species within the Acidimicrobia, Deinococci,

555 Deltaproteobacteria, Flavobacteriia, Gemmatimonadetes, Saprospirae and Sphingobacteriia

556 classes were identified as being potential CO oxidisers, indicating that the diversity of bacteria

557 capable of CO oxidation may be higher than currently realised. Analysis of the functional 558 diversity of $\mathrm{CO}$ oxidisers based on the coxL gene provided further support that some of these

559 candidates are indeed capable of $\mathrm{CO}$ oxidation. Four of the candidates identified by high

560 throughput sequencing of $16 \mathrm{~S}$ rRNA genes were Rhizobiales species and the majority of the

561 coxL sequences were most similar to those of Rhizobiales species, with five different clades

562 of coxL likely belonging to this order. It is therefore likely that these candidates (OTU90,

563 OTU269, OTU1705 and OTU72) are capable of CO oxidation. In addition, four species of

564 Burkholderiales (OTU87, OTU10, OTU36 and OTU20) were identified in the rRNA based

565 analyses. Interestingly, all of these OTUs were abundant in the phyllosphere at the time of

566 sampling, making up $0.12-10 \%$ of total reads in the original leaf wash samples. A cluster of

$567 \operatorname{coxL}$ homologues sequenced from the clone library were most similar to, but distinct from,

568 coxL genes from a Burkholderia species. It is possible that this coxL gene is from one of the

569 Burkholderiales candidates. In a parallel study investigating the degradation of 4-nitrophenol

570 by phyllosphere microbiota (Palmer et al., unpublished), the metagenome assembled

571 genomes (MAGs) of two species of Burkholderiales: an Actinomycetospora species and a

572 Variovorax species believed to be OTU13 and OTU10, respectively, were obtained. These

573 draft genomes contained the form II arrangement of cox genes, providing further evidence

574 that some abundant phyllosphere colonisers are likely to be capable of CO oxidation. 
576 indicate that CODH genes may be abundant within the phyllosphere microbial community.

577 Numerous CODH homologues were detected in all the phyllosphere metagenomes available.

578 Furthermore, when normalised with three housekeeping genes, it is estimated that up to $55 \%$

579 of the phyllosphere microbial community contained coxL gene homologues, or an average of

$58025 \%$ contained cox genes ( $\operatorname{coxL}, \operatorname{cox} M$ and $\operatorname{coxS}$ ), whereas only up to $25.9 \%$ contained mxaF gene homologues, or an average of $7.5 \%$ from both mxaF and mxal. Methanol metabolism is a well-known function of the phyllosphere due to the high availability of plant-produced methanol as a substrate on the leaf surface $[4,8,82]$. Therefore, these preliminary functional analyses provide an initial indication that $\mathrm{CO}$ oxidation has the potential to be an equally important and widespread function of phyllosphere microbiota. The estimated abundance of bacteria capable of $\mathrm{CO}$ oxidation in the phyllosphere in this study compliments recent estimates that $56 \%$ of soil bacteria are capable of $\mathrm{CO}$ oxidation [60]. It is possible that the phyllosphere and soil may harbour similar communities of CO-oxidising bacteria as these biomes seed one another by aerial dispersal, leaf fall, precipitation runoff and plant seedling inoculation. Due to the short sequences of the phyllosphere metagenome cox gene homologues which were available on MG-RAST, many of the CODH may be form II types of which the function is not fully known. Therefore, further research into the activity of form II $\mathrm{CODH}$ in $\mathrm{CO}$ oxidation, along with additional meta-omics investigations of phyllosphere communities, is necessary before the potential of phyllosphere microbiota to oxidise CO is understood.

The phyllosphere CO-oxidising isolate CO17, a member of the Phyllobacteriaceae, was most closely related to Mezorhizobium zhangyense [87]. Some Mesorhizobium species are already known to oxidise $\mathrm{CO}$ [48], and are common phyllosphere colonisers [88]. However, isolate CO17 and Mesorhizobium zhangyense do not cluster with other Mesorhizobium species (Fig. 5A). CO17 was also identified as an 'unclassified Phyllobacteriaceae' according to the RDP classifier [89] whereas Mesorhizobium zhangyense is not currently listed as a validated Mesorhizobium species in the LPSN [90], indicating that the taxonomic classification of both of these species requires further attention. Likewise, it is noteworthy that isolate CO17 was isolated from a CO enrichment culture where CO was the sole carbon source and 
606

607

608

609

610

611

612

613

614

615

616

617

618

619

620

621

622

\section{3}

624

625

626

627

628

629

630

631

632

633

634

635

636

assimilation of $\mathrm{CO}_{2}$ from $\mathrm{CO}$ oxidation remains to be determined; previously identified COoxidising Mesorhizobium species did not grow on $\mathrm{CO}$ as a carbon source.

\section{Conclusions}

Overall, this work demonstrates that the phyllosphere is host to diverse microorganisms capable of oxidising $\mathrm{CO}$. Some of the candidate CO-oxidising species identified here are abundant members of the phyllosphere microbial community and the preliminary functional analyses of phyllosphere communities indicate an average of $25 \%$ of bacterial community colonising the phyllosphere contain cox ( $\operatorname{coxL}$, $\operatorname{coxM}$ and $\operatorname{coxL}$ ) gene homologues and therefore may be capable of $\mathrm{CO}$ oxidation. Recent research has suggested that carboxydovores capable of $\mathrm{CO}$ oxidation at sub-atmospheric concentrations are abundant in both marine and soil samples $[59,60]$. As the leaf surface is a nutrient-poor environment with direct exposure to atmospheric $\mathrm{CO}$, we propose that $\mathrm{CO}$ oxidation may be a widespread function of the phyllosphere. Further work is required to establish the contribution of phyllosphere microbiota to the global CO cycling and mitigation of this toxic air pollutant.

\section{List of abbreviations}

ANOSIM: Analysis of similarities

CO: Carbon monoxide

CODH: Carbon monoxide dehydrogenase

GC: Gas chromatography

OTU: Operational taxonomic unit

PCR: Polymerase chain reaction

SIMPER: Similarity percentage analysis

\section{Declarations}

\section{Ethics approval and consent to participate}

Not applicable. 


\section{Consent for publication}

638 Not applicable.

639

640 Availability of data and material

641 The raw 16S rRNA gene sequences from Illumina MiSeq amplicon sequencing were deposited 642 at NCBI Sequence Read Archive (SRA) under the BioProject accession ID PRJNA699530. The 643 16S rRNA gene sequences of all CO-oxidising candidate species were deposited at NCBI 644 Genbank under the accession numbers MW596379-MW596407. All coxL genes from clone 645 libraries were deposited at NCBI Genbank under the accession numbers MW596683646 MW596710. The 16S rRNA gene of CO-oxidising isolate CO17 was submitted at NCBI Genbank 647 under the accession MW592845 whereas the assembled genome sequence was submitted 648 under the accession of JAFNIO000000000.

649

650 Competing interests

651 The authors declare that they have no competing interests.

652

\section{Funding}

654 This study was supported by the Natural Environment Research Council (NERC), Central 655 England NERC Training Alliance (CENTA) DTP.

656

\section{Author's contributions}

658 JLP designed the research studies, performed the laboratory experiments, analysed the data 659 and wrote the manuscript. SH assisted with the MiSeq library preparation and analysis of 660 data. EP assisted with data analysis. GDB designed the research studies and edited the 661 manuscript. HS conceived the project, designed the research studies and edited the 662 manuscript. All authors read and approved the final manuscript.

663

664 Acknowledgements

665 Genome sequencing was provided by MicrobesNG (http://www.microbesng.uk). We would 666 like to thank Dr. Eileen Kröber (Max Plank Institute for Marine Microbiology) for her 667 experimental support. 
Additional file 1: Figure S1. Diversity of coxL genes used in coxL gene primer design. The tree was deduced using the Neighbour-Joining algorithm implemented in ARB with PAM distance correction based on an alignment of nucleotide sequences [65]. Asterix $(*)$ indicates sequences which were not used in coxL primer design.

674

Additional file 2: Table S1. Modification of form I coxL gene primers. Mismatches identified in bases are indicated in red. Modifications made to bases in new primer pair are indicated in 677 green.

678

Additional file 3: Figure S2. Degradation of $\mathrm{CO}$ as a sole carbon source by holly and hawthorn leaf wash communities from heterotrophic enrichment cultures. Enrichment culture medium contained minimal media with 800_ppm CO and no additional carbon source. A) Woodland hawthorn (HtW) samples. B) Roadside hawthorn (HtR) samples. C) Woodland holly (HIW) samples. Spikes in CO concentration indicate where CO was added to the enrichment culture upon degradation to beyond the detectable limit (<50_ppm).

Additional file 4: Figure S3. Relative abundance of OTUs enriched in leaf wash cultures Samples shown in this figure represent leaf wash cultures which degraded 800 ppm CO, with yeast extract, four consecutive times during three sub-cultures. OTUs which contributed to $<2 \%$ of total reads on average in the CO enrichment group were placed in the 'Low abundance' category.

692

Additional file 5: Figure S4. Relative abundance of OTUs enriched with 800 ppm CO as a sole carbon source compared to control cultures without CO. Samples shown in this figure are those which successfully oxidised four spikes of 800 ppm CO as a sole carbon source after being sub-cultured from $\mathrm{CO}$ cultures which contained yeast extract as an additional carbon source. OTUs which contributed to $<2 \%$ of total reads on average in the $\mathrm{CO}$ enrichment group were placed in the 'Low abundance' category. 
700 Additional file 6: Figure S5. Bacterial OTUs identified as indicator species in leaf wash

701

702

703

704

705

706

707

708

709

710

711

712

713

714

715

716

717

718

719

720 cultures enriched with $800 \mathrm{ppm}$ CO with or without an additional carbon source. A) Leaf wash OTUs significantly enriched by CO in cultures containing 800 ppm CO plus yeast extract. B) OTUs from heterotrophic CO enrichment cultures which were enriched when sub-cultured into cultures containing 800 ppm CO as a sole carbon source. OTUs in red indicate those with $P<0.05$ and $\log _{2}$ fold change $>2$. OTUs in blue indicate those with $P<0.05$ and $\log _{2}$ fold change $=<2$. OTUs in grey indicate those with $\mathrm{P}>0.05$ and $\log _{2}$ fold change $<2$. OTUs in green indicate those with $\mathrm{P}>0.05$ and $\log _{2}$ fold change $>2$.

Additional file 7: Figure S6. Phylogeny of the RubisCO protein of CO-oxidising isolate CO17. Bona fide form I Rubisco amino acid sequences were identified using data from Nanba et al., [91]. Phylogeny of CO17 RubisCO and other form I RubisCO sequences was inferred using the Neighbor-Joining method [1] of amino acid sequences in MEGA7 [2]. The percentage of replicate trees in which the associated taxa clustered together in the bootstrap test (500 replicates) are shown next to the branches. The scale bar denotes the number of amino acid differences per site. 


\section{References}

722

723

724

725

726

727

728

729

730

731

732

733

734

735

736

737

738

739

740

741

742

743

744

745

746

747

748

749

750

751

752

753

754

755

756

757

758

759

760

761

762

763

764

765

1. Saitou N, Nei M: The neighbor-joining method - A new method for reconstructing phylogenetic trees. . Molecular Biology and Evolution 1987, 4(4):406-425.

2. Kumar S, Stecher G, Tamura K: MEGA7: Molecular Evolutionary Genetics Analysis version 7.0 for bigger datasets. Molecular Biology and Evolution 2016, 33(7):18701874.

3. Felsenstein J: Confidence limits on phylogenies - An approach using the bootstrap. Evolution 1985, 39(4):783-791.

4. Vorholt JA: Microbial life in the phyllosphere. Nature Reviews Microbiology 2012, 10(12):828-840.

5. Woodward F, Lomas M: Vegetation dynamics-simulating responses to climatic change. Biological Reviews 2004, 79(3):643-670.

6. Lindow SE, BrandI MT: Microbiology of the phyllosphere. Applied and Environmental Microbiology 2003, 69(4):1875-1883.

7. Redford AJ, Bowers RM, Knight R, Linhart Y, Fierer N: The ecology of the phyllosphere: geographic and phylogenetic variability in the distribution of bacteria on tree leaves. Environmental Microbiology 2010, 12(11):2885-2893.

8. Knief C, Delmotte N, Chaffron S, Stark M, Innerebner G, Wassmann R, von Mering C, Vorholt JA: Metaproteogenomic analysis of microbial communities in the phyllosphere and rhizosphere of rice. ISME Journal 2012, 6(7):1378-1390.

9. Kembel SW, Mueller RC: Plant traits and taxonomy drive host associations in tropical phyllosphere fungal communities. Botany 2014, 92(4).

10. Whipps JM, Hand P, Pink D, Bending GD: Phyllosphere microbiology with special reference to diversity and plant genotype. Journal of Applied Microbiology 2008, 105(6):1744-1755.

11. Leveau JH, Lindow SE: Appetite of an epiphyte: quantitative monitoring of bacterial sugar consumption in the phyllosphere. Proceedings of the National Academy of Sciences 2001, 98(6):3446-3453.

12. Remus-Emsermann MN, Tecon R, Kowalchuk GA, Leveau JH: Variation in local carrying capacity and the individual fate of bacterial colonizers in the phyllosphere. The ISME Journal 2012, 6(4):756-765.

13. Knief C, Ramette A, Frances L, Alonso-Blanco C, Vorholt JA: Site and plant species are important determinants of the Methylobacterium community composition in the plant phyllosphere. ISME Journal 2010, 4(6):719-728.

14. Sandhu A, Halverson $\sqcup$, Beattie GA: Identification and genetic characterization of phenol-degrading bacteria from leaf microbial communities. Microbial Ecology 2009, 57(2):276-285.

15. Scheublin TR, Leveau JHJ: Isolation of Arthrobacter species from the phyllosphere and demonstration of their epiphytic fitness. Microbiologyopen 2013, 2(1):205-213.

16. Jindachot W, Treesubsuntorn C, Thiravetyan P: Effect of individual/co-culture of native Phyllosphere organisms to enhance Dracaena sanderiana for benzene phytoremediation. Water, Air, \& Soil Pollution 2018, 229(3):80.

17. Yutthammo C, Thongthammachat N, Pinphanichakarn $\mathrm{P}$, Luepromchai E: Diversity and activity of PAH-degrading bacteria in the phyllosphere of ornamental plants. Microbial Ecology 2010, 59(2):357-368. 
18. Nadalig T, Farhan UI Haque M, Roselli S, Schaller H, Bringel F, Vuilleumier S: Detection and isolation of chloromethane-degrading bacteria from the Arabidopsis thaliana phyllosphere, and characterization of chloromethane utilization genes. FEMS Microbiology Ecology 2011, 77(2):438-448.

19. Crombie AT, Larke-Mejia NL, Emery H, Dawson R, Pratscher J, Murphy GP, McGenity TJ, Murrell JC: Poplar phyllosphere harbors disparate isoprene-degrading bacteria. Proceedings of the National Academy of Sciences USA 2018, 115(51):13081-13086.

20. Carrión O, Gibson L, Elias DM, McNamara NP, van Alen TA, Op den Camp HJ, Supramaniam CV, McGenity TJ, Murrell JC: Diversity of isoprene-degrading bacteria in phyllosphere and soil communities from a high isoprene-emitting environment: a Malaysian oil palm plantation. Microbiome 2020, 8:1-13.

21. Imperato V, Kowalkowski L, Portillo-Estrada M, Gawronski SW, Vangronsveld J, Thijs $\mathrm{S}$ : Characterisation of the Carpinus betulus L. phyllomicrobiome in urban and forest areas. Frontiers in Microbiology 2019, 10.

22. Lelieveld J, Evans JS, Fnais M, Giannadaki D, Pozzer A: The contribution of outdoor air pollution sources to premature mortality on a global scale. Nature 2015, 525(7569):367-371.

23. Liu H, Tian Y, Xiang X, Li M, Wu Y, Cao Y, Juan J, Song J, Wu T, Hu Y: Association of short-term exposure to ambient carbon monoxide with hospital admissions in China. Scientific reports 2018, 8(1):1-7.

24. Daniel JS, Solomon S: On the climate forcing of carbon monoxide. Journal of Geophysical Research-Atmospheres 1998, 103(D11):13249-13260.

25. Reeves CE, Penkett SA, Bauguitte S, Law KS, Evans MJ, Bandy BJ, Monks PS, Edwards $\mathrm{GD}$, Phillips $\mathrm{G}$, Barjat $\mathrm{H}$ et al: Potential for photochemical ozone formation in the troposphere over the North Atlantic as derived from aircraft observations during ACSOE. Journal of Geophysical Research-Atmospheres 2002, 107(D23).

26. Westberg K, Cohen N, Wilson KW: Carbon monoxide - its role in photochemical smog formation. Science 1971, 171(3975):1013-1015.

27. Badr O, Probert S: Carbon monoxide concentration in the Earth's atmosphere. Applied Energy 1994, 49(2):99-143.

28. Stubbins A, Uher G, Law CS, Mopper K, Robinson C, Upstill-Goddard RC: Open-ocean carbon monoxide photoproduction. Deep Sea Research Part II: Topical Studies in Oceanography 2006, 53(14-16):1695-1705.

29. Seiler W, Giehl H: Influence of plants on atmospheric carbon-monoxide. Geophysical Research Letters 1977, 4(8):329-332.

30. Tarr MA, Miller WL, Zepp RG: Direct carbon-monoxide photoproduction from plant matter. Journal of Geophysical Research-Atmospheres 1995, 100(D6):11403-11413.

31. Khalil MAK, Rasmussen RA: Global decrease in atmospheric carbon-monoxide concentration. Nature 1994, 370(6491):639-641.

32. King GM, Weber CF: Distribution, diversity and ecology of aerobic CO-oxidizing bacteria. Nature Reviews Microbiology 2007, 5(2):107-118.

33. DEFRA: Air Pollution in the UK 2014. Department for Environment, Food and Rural Affairs 2014, [Online] Available at: https://ukair.defra.gov.uk/library/annualreport/viewonline?year=2014 issue_1\#report_pdf.

34. Ou-Yang C-F, Lin N-H, Lin C-C, Wang S-H, Sheu G-R, Lee C-T, Schnell RC, Lang PM, Kawasato $\mathrm{T}$, Wang J-L: Characteristics of atmospheric carbon monoxide at a high- 
mountain background station in East Asia. Atmospheric Environment 2014, 89:613622.

35. Khalil M, Rasmussen R: Carbon monoxide in the earth's atmosphere: increasing trend. Science 1984, 224(4644):54-56.

36. Novelli P, Masarie K, Lang P: Distributions and recent changes of carbon monoxide in the lower troposphere. Journal of Geophysical Research: Atmospheres 1998, 103(D15):19015-19033.

37. Xue $M$, Wang $Y S$, Sun $Y$, Hu B, Wang MX: Measurement on the atmospheric CO concentration in Beijing. Environmental Science 2006, 27(2):200-206.

38. Badr O, Probert S: Sinks and environmental impacts for atmospheric carbon monoxide. Applied Energy 1995, 50(4):339-372.

39. King GM: Attributes of atmospheric carbon monoxide oxidation by Maine forest soils. Applied and Environmental Microbiology 1999, 65(12):5257-5264.

40. Conte L, Szopa S, Seferian R, Bopp L: The oceanic cycle of carbon monoxide and its emissions to the atmosphere. Biogeosciences 2019, 16(4):881-902.

41. Xie HX, Zafiriou OC, Umile TP, Kieber DJ: Biological consumption of carbon monoxide in Delaware Bay, NW Atlantic and Beaufort Sea. Marine Ecology Progress Series 2005, 290:1-14.

42. Tolli JD, Taylor CD: Biological CO oxidation in the Sargasso Sea and in Vineyard Sound, Massachusetts. Limnology and Oceanography 2005, 50(4):1205-1212.

43. Moran MA, Buchan A, Gonzalez JM, Heidelberg JF, Whitman WB, Kiene RP, Henriksen JR, King GM, Belas R, Fuqua C et al: Genome sequence of Silicibacter pomeroyi reveals adaptations to the marine environment. Nature 2004, 432(7019):910-913.

44. Meyer O, Schlegel HG: Biology of aerobic carbon-monoxide oxidizing bacteria. Annual Review of Microbiology 1983, 37:277-310.

45. Lorite MJ, Tachil J, Sanjuan J, Meyer O, Bedmar EJ: Carbon monoxide dehydrogenase activity in Bradyrhizobium japonicum. Applied and Environmental Microbiology 2000, 66(5):1871-1876.

46. Hogendoorn C, Pol A, Picone N, Cremers G, van Alen TA, Gagliano AL, Jetten MS, D'Alessandro W, Quatrini P, den Camp HJO: Hydrogen and Carbon MonoxideUtilizing Kyrpidia spormannii Species From Pantelleria Island, Italy. Frontiers in Microbiology 2020, 11.

47. Cunliffe $M$ : Correlating carbon monoxide oxidation with cox genes in the abundant Marine Roseobacter Clade. ISME Journal 2011, 5(4):685-691.

48. King GA: Molecular and culture-based analyses of aerobic carbon monoxide oxidizer diversity. Applied and Environmental Microbiology 2003, 69(12):7257-7265.

49. Tolli JD, Sievert SM, Taylor CD: Unexpected diversity of bacteria capable of carbon monoxide oxidation in a coastal marine environment, and contribution of the Roseobacter-associated clade to total CO oxidation. Applied and Environmental Microbiology 2006, 72(3):1966-1973.

50. Weber CF, King GM: Volcanic soils as sources of novel CO-oxidizing Paraburkholderia and Burkholderia: Paraburkholderia hiiakae sp nov., Paraburkholderia metrosideri sp nov., Paraburkholderia paradisi sp nov., Paraburkholderia peleae sp nov., and Burkholderia alpina sp nov a member of the Burkholderia cepacia complex. Frontiers in Microbiology 2017, 8. 
51. Park SW, Hwang EH, Park H, Kim JA, Heo J, Lee KH, Song T, Kim E, Ro YT, Kim SW et al: Growth of mycobacteria on carbon monoxide and methanol. Journal of Bacteriology 2003, 185(1):142-147.

52. Yang J, Zhou EM, Jiang HC, Li WJ, Wu G, Huang LQ, Hedlund BP, Dong HL: Distribution and diversity of aerobic carbon monoxide-oxidizing bacteria in geothermal springs of China, the Philippines, and the United States. Geomicrobiology Journal 2015, 32(10):903-913.

53. Weber CF, King GM: Distribution and diversity of carbon monoxide-oxidizing bacteria and bulk bacterial communities across a succession gradient on a Hawaiian volcanic deposit. Environmental Microbiology 2010, 12(7):1855-1867.

54. Wu XH, Ge TD, Hu YJ, Wei XM, Chen L, Whiteley AS, Wu JS: Abundance and diversity of carbon monoxide dehydrogenase genes from BMS clade bacteria in different vegetated soils. European Journal of Soil Biology 2017, 81:94-99.

55. Hardy KR, King GM: Enrichment of high-affinity CO oxidizers in Maine forest soil. Applied and Environmental Microbiology 2001, 67(8):3671-3676.

56. Lalonde I, Constant P: Identification of unknown carboxydovore bacteria dominant in deciduous forest soil via succession of bacterial communities, coxL genotypes, and carbon monoxide oxidation activity in soil microcosms. Applied and Environmental Microbiology 2016, 82(4):1324-1333.

57. Venter JC, Remington K, Heidelberg JF, Halpern AL, Rusch D, Eisen JA, Wu DY, Paulsen I, Nelson KE, Nelson W et al: Environmental genome shotgun sequencing of the Sargasso Sea. Science 2004, 304(5667):66-74.

58. Hernández M, Vera-Gargallo B, Calabi-Floody M, King GM, Conrad R, Tebbe CC: Reconstructing genomes of carbon monoxide oxidisers in volcanic deposits including members of the class Ktedonobacteria. Microorganisms 2020, 8(12):1880.

59. Cordero PR, Bayly K, Leung PM, Huang C, Islam ZF, Schittenhelm RB, King GM, Greening C: Atmospheric carbon monoxide oxidation is a widespread mechanism supporting microbial survival. The ISME Journal 2019, 13(11):2868-2881.

60. Bay SK, Dong X, Bradley JA, Leung PM, Grinter R, Jirapanjawat T, Arndt SK, Cook PL, LaRowe DE, Nauer PA: Trace gas oxidizers are widespread and active members of soil microbial communities. Nature Microbiology 2021:1-11.

61. Atamna-Ismaeel N, Finkel OM, Glaser F, Sharon I, Schneider R, Post AF, Spudich JL, von Mering C, Vorholt JA, lluz D et al: Microbial rhodopsins on leaf surfaces of terrestrial plants. Environmental Microbiology 2011, 14(1):140-146.

62. Kanagawa T, Dazai M, Fukuoka S: Degradation of organo-phosphorus wastes .2. degradation of 0,0-dimethyl phosphorodithioate by Thiobacillus-thioparus TK-1 and Pseudomonas AK-2. Agricultural and Biological Chemistry 1982, 46(10):25712578.

63. Caporaso JG, Lauber CL, Walters WA, Berg-Lyons D, Huntley J, Fierer N, Owens SM, Betley J, Fraser L, Bauer $M$ et al: Ultra-high-throughput microbial community analysis on the Illumina HiSeq and MiSeq platforms. ISME Journal 2012, 6(8):16211624.

64. Chen IMA, Chu K, Palaniappan K, Pillay M, Ratner A, Huang JH, Huntemann M, Varghese N, White JR, Seshadri R et al: IMG/M v.5.0: an integrated data management and comparative analysis system for microbial genomes and microbiomes. Nucleic Acids Research 2019, 47(D1):D666-D677. 
65. Ludwig W, Strunk O, Westram R, Richter L, Meier H, Yadhukumar, Buchner A, Lai T, Steppi $S$, Jobb $G$ et al: ARB: a software environment for sequence data. Nucleic Acids Research 2004, 32(4):1363-1371.

66. Weisburg WG, Barns SM, Pelletier DA, Lane DJ: 16S ribosomal DNA amplification for phylogenetic study. Journal of Bacteriology 1991, 173(2):697-703.

67. Bolger AM, Lohse $M$, Usadel B: Trimmomatic: a flexible trimmer for Illumina sequence data. Bioinformatics 2014, 30(15):2114-2120.

68. Bankevich A, Nurk S, Antipov D, Gurevich AA, Dvorkin M, Kulikov AS, Lesin VM, Nikolenko SI, Pham S, Prjibelski AD et al: SPAdes: a new genome assembly algorithm and its applications to single-cell sequencing. Journal of Computational Biology 2012, 19(5):455-477.

69. Aziz RK, Bartels D, Best AA, DeJongh M, Disz T, Edwards RA, Formsma K, Gerdes S, Glass EM, Kubal $M$ et al: The RAST server: Rapid annotations using subsystems technology. BMC Genomics 2008, 9.

70. Edgar RC: Search and clustering orders of magnitude faster than BLAST. Bioinformatics 2010, 26(19):2460-2461.

71. Edgar RC, Haas BJ, Clemente JC, Quince C, Knight R: UCHIME improves sensitivity and speed of chimera detection. Bioinformatics 2011, 27(16):2194-2200.

72. Caporaso JG, Kuczynski J, Stombaugh J, Bittinger K, Bushman FD, Costello EK, Fierer N, Pena AG, Goodrich JK, Gordon Jl et al: QIIME allows analysis of high-throughput community sequencing data. Nature Methods 2010, 7(5):335-336.

73. McDonald D, Price MN, Goodrich J, Nawrocki EP, DeSantis TZ, Probst A, Andersen GL, Knight R, Hugenholtz P: An improved Greengenes taxonomy with explicit ranks for ecological and evolutionary analyses of bacteria and archaea. ISME Journal 2012, 6(3):610-618.

74. Dixon P: VEGAN, a package of R functions for community ecology. Journal of Vegetation Science 2003, 14(6):927-930.

75. Clarke K, Gorley R: PRIMER: Getting started with v6. PRIMER-E Ltd: Plymouth, UK 2005.

76. Love MI, Huber W, Anders S: Moderated estimation of fold change and dispersion for RNA-seq data with DESeq2. Genome Biology 2014, 15(12):550.

77. Blighe K: EnhancedVolcano: Publication-ready volcano plots with enhanced colouring and labeling. R package version 1.2. 0. In.; 2019.

78. Johnson M, Zaretskaya I, Raytselis Y, Merezhuk Y, McGinnis S, Madden TL: NCBI BLAST: a better web interface. Nucleic Acids Research 2008, 36(suppl_2):W5-W9.

79. Edgar RC: MUSCLE: multiple sequence alignment with high accuracy and high throughput. Nucleic Acids Research 2004, 32(5):1792-1797.

80. Douglas GM, Maffei VJ, Zaneveld J, Yurgel SN, Brown JR, Taylor CM, Huttenhower C, Langille MG: PICRUSt2: An improved and extensible approach for metagenome inference. BioRxiv 2019:672295.

81. Keegan KP, Glass EM, Meyer F: MG-RAST, a metagenomics service for analysis of microbial community structure and function. In: Microbial environmental genomics (MEG). Springer; 2016: 207-233.

82. Delmotte N, Knief C, Chaffron S, Innerebner G, Roschitzki B, Schlapbach R, von Mering $C$, Vorholt JA: Community proteogenomics reveals insights into the physiology of phyllosphere bacteria. Proceedings of the National Academy of Sciences USA 2009, 106(38):16428-16433. 
83. Kembel SW, O'Connor TK, Arnold HK, Hubbell SP, Wright SJ, Green JL: Relationships between phyllosphere bacterial communities and plant functional traits in a neotropical forest. Proceedings of the National Academy of Sciences USA 2014, 111(38):13715-13720.

84. Dwivedi VD, Arora S, Kumar A, Mishra SK: Computational analysis of xanthine dehydrogenase enzyme from different source organisms. Network Modeling Analysis in Health Informatics and Bioinformatics 2013, 2(4):185-189.

85. Douglas GM, Maffei VJ, Zaneveld J, Yurgel SN, Brown JR, Taylor CM, Huttenhower C, Langille MG: PICRUSt2: An improved and customizable approach for metagenome inference. BioRxiv 2020:672295.

86. Lyons CM, Justin P, Colby J, Williams E: Isolation, characterization and autotrophic metabolism of a moderately thermophilic carboxydobacterium, pseudomonasThermocarboxydovorans sp-nov. Journal of General Microbiology 1984, 130(MAY):1097-1105.

87. Xu L, Zhang Y, Mohamad OA, Jiang C, Friman V-P: Mesorhizobium zhangyense sp. nov., isolated from wild Thermopsis lanceolate in northwestern China. Archives of microbiology 2018, 200(4):603-610.

88. Vogel C, Innerebner G, Zingg J, Guder J, Vorholt JA: Forward genetic in planta screen for identification of plant-protective traits of Sphingomonas sp. strain Fr1 against Pseudomonas syringae DC3000. Applied and Environmental Microbiology 2012, 78(16):5529-5535.

89. Wang Q, Garrity GM, Tiedje JM, Cole JR: Naive Bayesian classifier for rapid assignment of rRNA sequences into the new bacterial taxonomy. Applied and environmental microbiology 2007, 73(16):5261-5267.

90. Parte AC, Sardà Carbasse J, Meier-Kolthoff JP, Reimer LC, Göker M: List of Prokaryotic names with Standing in Nomenclature (LPSN) moves to the DSMZ. International Journal of Systematic and Evolutionary Microbiology 2020:ijsem004332.

91. Nanba K, King G, Dunfield K: Analysis of facultative lithotroph distribution and diversity on volcanic deposits by use of the large subunit of ribulose $1,5-$ bisphosphate carboxylase/oxygenase. Applied and Environmental Microbiology 2004, 70(4):2245-2253. 


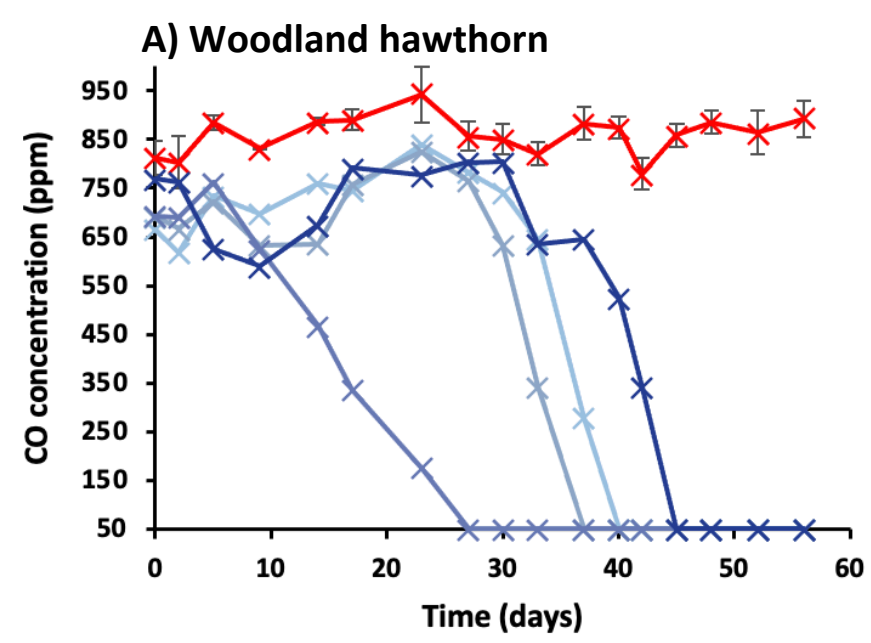

$\rightarrow$ Negative $\leftarrow$ HtW1 $\leftarrow$ HtW2 $\leftarrow$ HtW3 $\leftarrow$ HtW4

C) Woodland holly

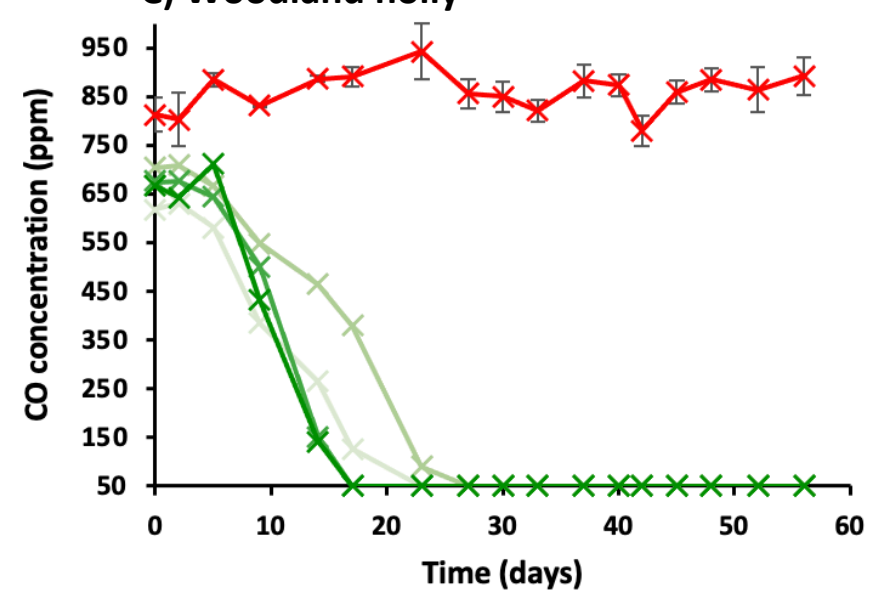

$\leftarrow$ Negative $\longrightarrow$ HIW1 $x$ HIW2 $\leftarrow$ HIW3 $\leftarrow$ HIW4
B) Roadside hawthorn

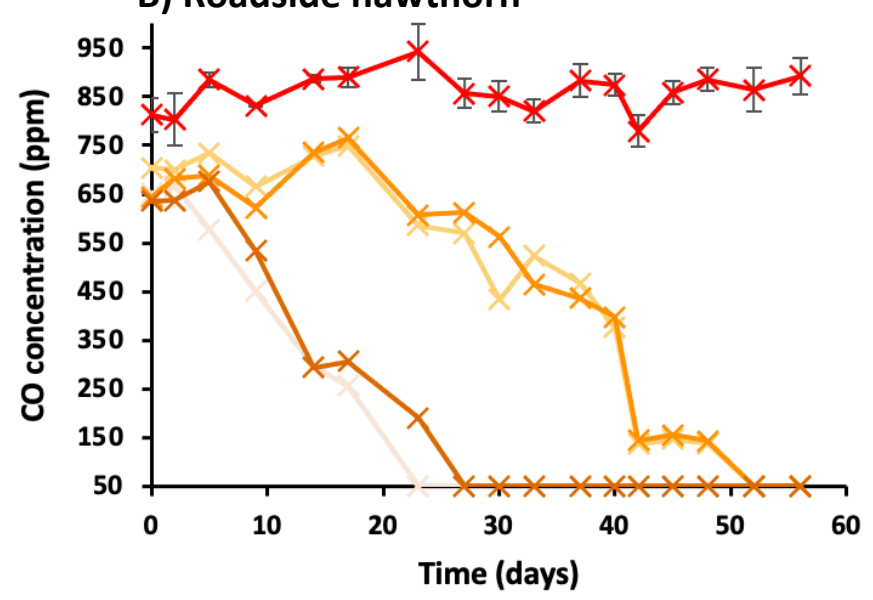

$\leftarrow$ Negative $\longrightarrow$ HtR1 $\leftarrow$ HtR2 $\leftarrow$ HtR3 $\leftarrow$ HtR4

D)

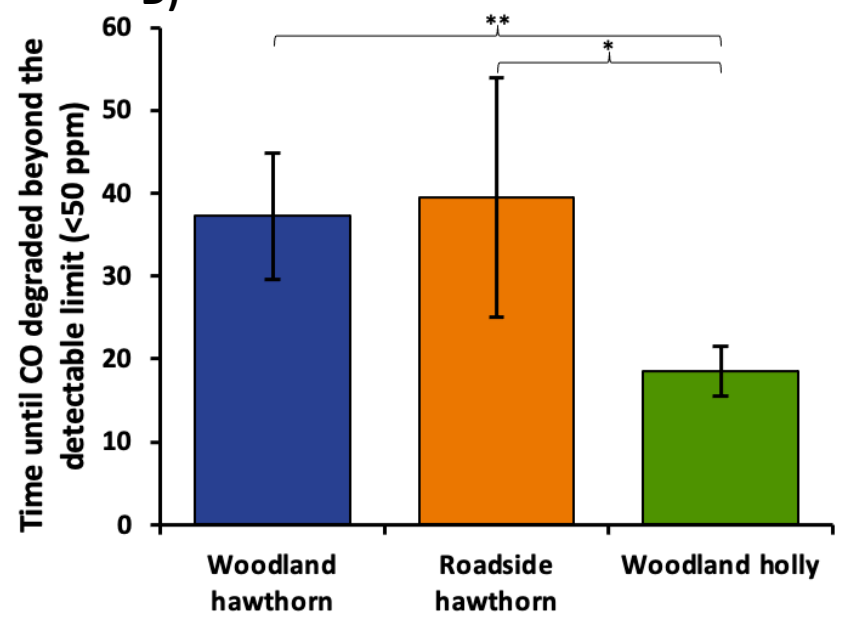

Sample type

Figure 1: Degradation of $\mathrm{CO}$ by holly and hawthorn leaf wash communities cultivated in media with added yeast extract. Enrichment culture medium contained $800 \_p p m ~ C O$ with $0.25 \mathrm{~g} / \mathrm{L}$ yeast extract. A) Woodland hawthorn (HtW) samples. B) Roadside hawthorn (HtR) samples. C) Woodland holly (HIW) samples. D) Average time taken for CO degradation between sample types $(* *=p$-value of $<0.001, *=p$-value of $<0.05$, error bars indicate standard deviation). Negative controls $(n=4)$ did not contain leaf wash as an inoculum. CO concentrations displayed in this figure are those of enrichment cultures where original leaf wash samples were used as an inoculum. $\mathrm{CO}$ oxidation data from subsequent sub-cultures is not shown. 

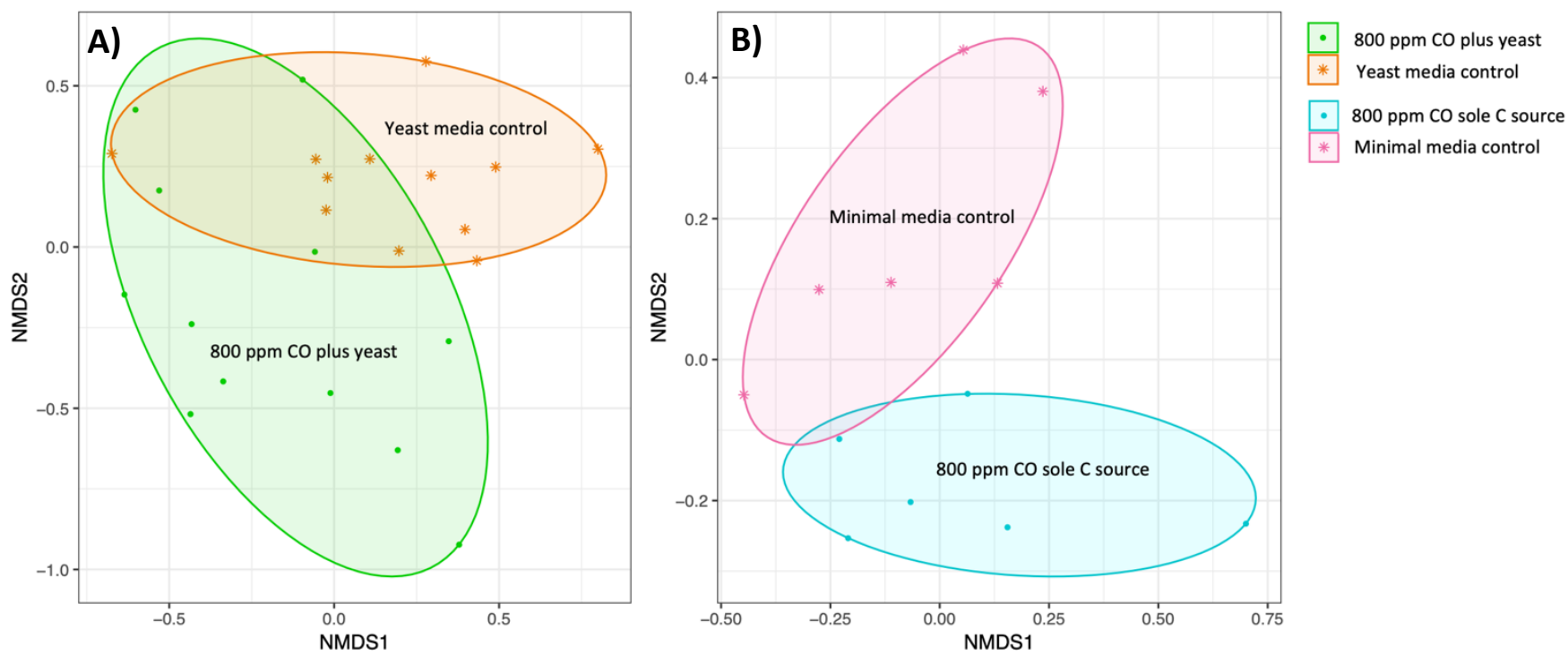

Figure 2: MDS ordination of variation between bacterial OTUs of $\mathrm{CO}$ enrichment culture samples and nosubstrate control incubations. MDS analyses were derived from Bray-Curtis similarity matrices constructed with relative read abundance data of OTUs present in each sample. A) Variation between leaf wash samples cultivated with or without $800 \mathrm{ppm}$ CO with the addition of another carbon source (yeast extract). B) Variation between subcultures from leaf wash samples cultivated with $800 \mathrm{ppm}$ CO plus yeast extract which were then able to oxidise $800 \mathrm{ppm} \mathrm{CO}$ as a sole carbon source and no-substrate controls. 
Table 1: Candidate CO-oxidising OTUs based on SIMPER analyses. Values represent relative abundance. Where Greengenes assignments are indicated, $o_{-}=$order, $f_{-}=$family, $g_{-}=$genus and $s_{-}=$species. Where values for unenriched leaf wash samples are indicated, $\mathrm{HtW}=$ woodland hawthorn samples, $\mathrm{HtR}=$ roadside hawthorn samples and $\mathrm{HIW}=$ woodland holly samples.

Unenriched leaf wash

\begin{tabular}{|c|c|c|c|c|c|c|c|c|}
\hline & OTU ID & $\begin{array}{l}\text { Contribution to } \\
\text { dissimilarity (\%) }\end{array}$ & Greengenes assignment & $\mathrm{CO}(\%)$ & Control (\%) & $\mathrm{HtW}(\%)$ & $\mathrm{HtR}(\%)$ & $\begin{array}{l}\text { HIW } \\
(\%)\end{array}$ \\
\hline \multirow{5}{*}{ 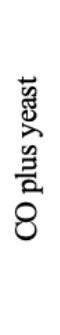 } & OTU34 & 6.38 & f_Xanthomonadaceae; g_Dokdonella & 8.004 & 3.425 & 0 & 0.006 & 0 \\
\hline & OTU792 & 4.59 & f_Micrococcaceae; g_Arthrobacter & 6.693 & 0.140 & 0 & 0 & 0 \\
\hline & OTU9 & 2.38 & g_Pseudomonas; s__viridiflava & 3.166 & 0.624 & 1.475 & 0.108 & 0.35 \\
\hline & OTU36 & 1.12 & o_Burkholderiales; f_Oxalobacteraceae & 1.611 & 0.087 & 0.164 & 0.403 & 1.294 \\
\hline & OTU128 & 1.09 & $\begin{array}{l}\text { f_Sphingobacteriaceae; } \\
\text { g__Sphingobacterium }\end{array}$ & 1.578 & 0.001 & 0 & 0 & 0 \\
\hline \multirow{9}{*}{ 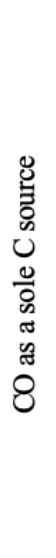 } & OTU97 & 5.03 & f_Xanthomonadaceae; $g \_$Rhodanobacter & 8.217 & 0.444 & 0.003 & 0.000 & 0.039 \\
\hline & OTU72 & 3.31 & o_Rhizobiales; f_Phyllobacteriaceae & 5.789 & 0.850 & 0.006 & 0.003 & 0.006 \\
\hline & OTU121 & 3.17 & o__Xanthomonadales; f_Xanthomonadacea & e3.898 & 2.230 & 0.000 & 0.003 & 0.000 \\
\hline & OTU90 & 3.06 & o_Rhizobiales; f_Bradyrhizobiaceae & 5.285 & 0.178 & 0.000 & 0.000 & 0.003 \\
\hline & OTU33 & 2.62 & g_Caulobacter; s_henricii & 4.470 & 0.065 & 0.003 & 0.000 & 0.000 \\
\hline & OTU5 & 2.05 & f_Xanthomonadaceae; g_Luteimonas & 3.478 & 0.059 & 0.003 & 0.008 & 0.047 \\
\hline & OTU194 & 1.34 & g_Nocardioides; s_plantarum & 2.261 & 0.000 & 0.069 & 0.014 & 0.022 \\
\hline & OTU10 & 1.11 & o_Burkholderiales; f_Comamonadaceae & 2.163 & 0.374 & 4.186 & 10.122 & 2.150 \\
\hline & OTU57 & 0.84 & g_Sphingopyxis; s_alaskensis & 1.533 & 0.311 & 0.000 & 0.000 & 0.008 \\
\hline
\end{tabular}

1009

1010

1011

1012

1013

1014

1015

1016 
Table 2: Candidate CO-oxidising OTUs indicated by those which showed the most significant log2 fold change between $\mathrm{CO}$ enrichment cultures and controls. Values represent relative abundance. Where greengenes assignments are indicated, $\mathrm{o}_{-}=$order, $\mathrm{f}_{-}=$family, $\mathrm{g}_{-}=$genus and $\mathrm{s}_{-}=$species. Where values for unenriched leaf wash samples are indicated, $\mathrm{HtW}=$ woodland hawthorn samples, $\mathrm{HtR}=$ roadside hawthorn samples and $\mathrm{HIW}=$ woodland holly samples.

\begin{tabular}{|c|c|c|c|c|c|c|c|c|c|}
\hline & \multirow{2}{*}{ OTU ID } & \multirow{2}{*}{ Greengenes assignment } & \multirow{2}{*}{$\mathrm{CO}(\%)$} & & \multicolumn{3}{|c|}{ Unenriched leaf wash } \\
\hline & & & & $\begin{array}{c}\text { Control } \\
(\%)\end{array}$ & $\begin{array}{l}\text { Log2 fold } \\
\text { change }\end{array}$ & P-value & HtW (\%) & $\operatorname{HtR}(\%)$ & HIW (\%) \\
\hline \multirow{14}{*}{ 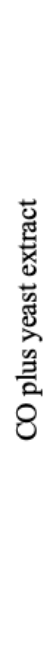 } & OTU792 & f_Micrococcaceae; g_Arthrobacter & 6.689 & 0.140 & 4.836 & 0.000 & 0.000 & 0.000 & 0.000 \\
\hline & OTU325 & o_Saprospirales; f_Chitinophagaceae & 0.265 & 0.003 & 3.768 & 0.008 & 0.000 & 0.000 & 0.000 \\
\hline & OTU114 & c_Deltaproteobacteria; o_Myxococcales & 0.311 & 0.015 & 3.302 & 0.002 & 0.000 & 0.000 & 0.003 \\
\hline & OTU355 & c_Gemmatimonadetes; o_Ellin5290 & 0.161 & 0.010 & 3.234 & 0.009 & 0.000 & 0.000 & 0.000 \\
\hline & OTU351 & o_Rhodospirillales; f_Rhodospirillaceae & 0.120 & 0.006 & 3.003 & 0.015 & 0.000 & 0.000 & 0.000 \\
\hline & OTU4556 & f_Sphingobacteriaceae; g_Pedobacter & 0.023 & 0.001 & 2.971 & 0.026 & 0.000 & 0.000 & 0.000 \\
\hline & OTU279 & o_Saprospirales; f_Chitinophagaceae & 0.683 & 0.000 & 2.487 & 0.000 & 0.000 & 0.000 & 0.000 \\
\hline & OTU5 & f_Xanthomonadaceae; g_Luteimonas & 0.467 & 0.104 & 2.419 & 0.027 & 0.003 & 0.008 & 0.047 \\
\hline & OTU423 & o_Rhodospirillales; f_Acetobacteraceae & 0.044 & 0.004 & 2.372 & 0.048 & 0.000 & 0.000 & 0.000 \\
\hline & OTU67 & f_Flavobacteriaceae; g_Flavobacterium & 0.377 & 0.017 & 2.348 & 0.020 & 0.003 & 0.003 & 0.003 \\
\hline & OTU235 & o_Saprospirales; f_Chitinophagaceae & 0.097 & 0.015 & 2.058 & 0.040 & 0.000 & 0.000 & 0.000 \\
\hline & OTU87 & o_Burkholderiales; f_Comamonadaceae & 2.066 & 0.730 & 1.818 & 0.038 & 0.356 & 0.242 & 0.508 \\
\hline & OTU20 & g_Janthinobacterium; s_lividum & 0.156 & 0.024 & 1.741 & 0.003 & 0.217 & 0.381 & 0.128 \\
\hline & OTU62 & f_Deinococcaceae;g_Deinococcus & 0.344 & 0.183 & 1.536 & 0.003 & 0.000 & 0.022 & 0.006 \\
\hline \multirow{9}{*}{ 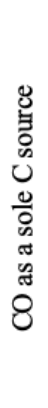 } & OTU267 & o_Rhodobacterales; f_Hyphomonadaceae & 0.617 & 0.006 & 4.603 & 0.007 & 0.000 & 0.000 & 0.000 \\
\hline & OTU90 & o_Rhizobiales; f_Bradyrhizobiaceae & 5.285 & 0.178 & 4.162 & 0.002 & 0.000 & 0.000 & 0.003 \\
\hline & OTU269 & f_Hyphomicrobiaceae; g_Rhodoplanes & 0.222 & 0.000 & 4.136 & 0.021 & 0.000 & 0.000 & 0.000 \\
\hline & OTU13 & f_Pseudonocardiaceae; g_Actinomycetospora & 0.200 & 0.000 & 4.043 & 0.023 & 1.989 & 1.372 & 4.331 \\
\hline & OTU359 & o_Acidimicrobiales; f_Microthrixaceae & 0.661 & 0.011 & 3.864 & 0.010 & 0.000 & 0.000 & 0.000 \\
\hline & OTU33 & g_Caulobacter; s_henricii & 4.470 & 0.065 & 3.735 & 0.010 & 0.003 & 0.000 & 0.000 \\
\hline & OTU355 & c_Gemmatimonadetes; o_Ellin5290 & 0.126 & 0.000 & 3.654 & 0.039 & 0.000 & 0.000 & 0.000 \\
\hline & OTU5 & f_Xanthomonadaceae; g_Luteimonas & 3.478 & 0.059 & 3.52 & 0.037 & 0.003 & 0.008 & 0.047 \\
\hline & OTU1705 & f_Hyphomicrobiaceae; g_Devosia & 0.539 & 0.011 & 3.33 & 0.034 & 0.000 & 0.000 & 0.003 \\
\hline
\end{tabular}




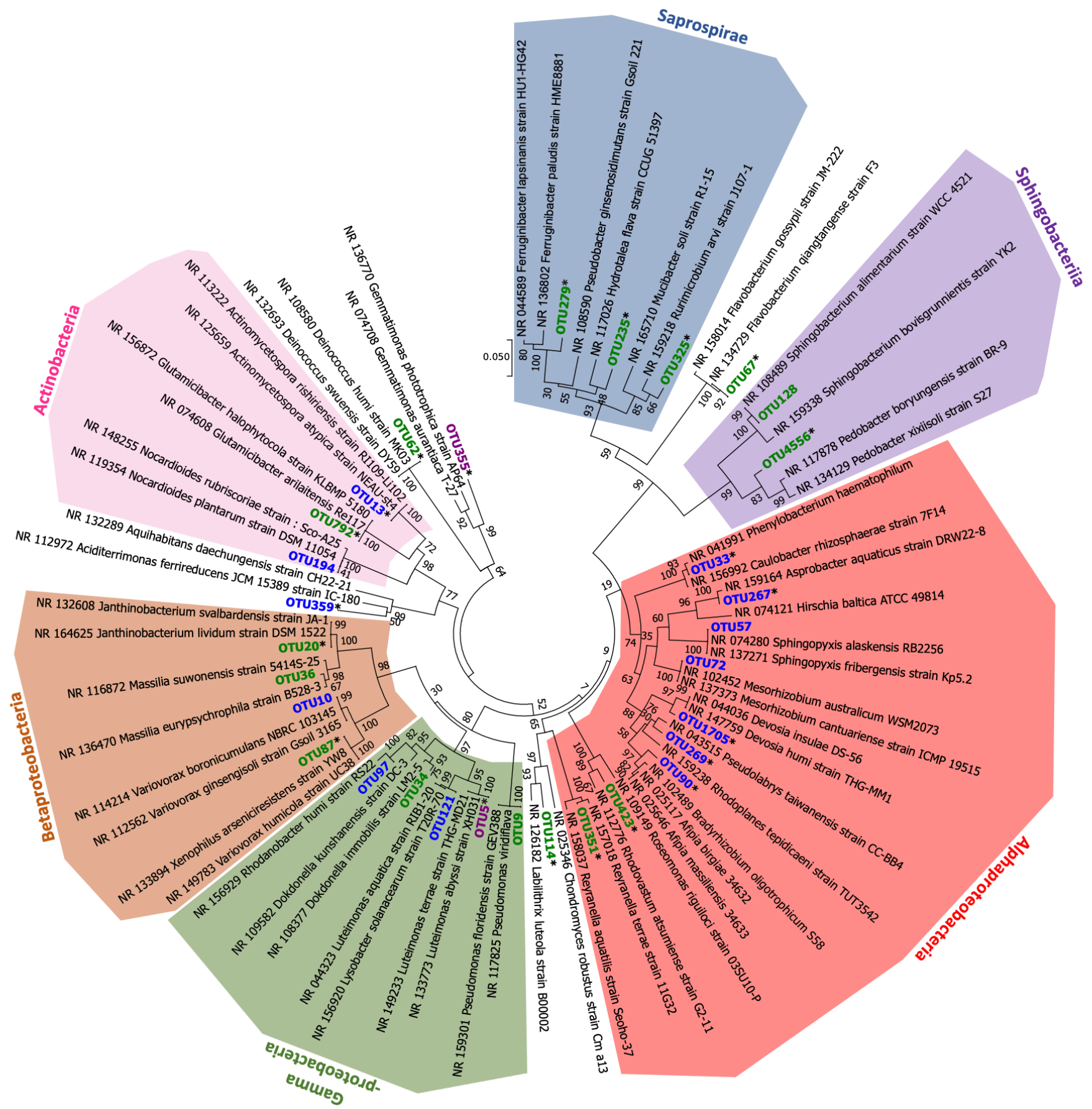

Figure 3: 16S rRNA phylogeny of candidate CO-oxidising bacteria from phyllosphere samples. OTUs were identified as potential CO-oxidising species from SIMPER and log2 fold change analyses between leaf wash samples cultivated with $\mathrm{CO}$ and substrate controls without $\mathrm{CO}$. OTUs indicated in green are those which were enriched in cultures where yeast extract was provided as a carbon source in addition to CO. OTUs indicated in blue are those which were enriched in cultures where $\mathrm{CO}$ was the sole carbon source. OTUs indicated in purple are those which were enriched in cultures where yeast extract was provided in addition to $\mathrm{CO}$ as well as $\mathrm{CO}$ as a sole carbon source. OTUs indicated with an Asterisk $(*)$ are those which had a significant ( $p$-value $=<0.05) \log$ fold change between $\mathrm{CO}$ enrichment cultures and the control group without $\mathrm{CO}$. Phylogeny of OTUs and BLAST hits was inferred using the Neighbor-Joining method [1] of nucleotide sequences in MEGA7 [2]. The percentage of replicate trees in which the associated taxa clustered together in the bootstrap [3] test (500 replicates) are shown next to the branches. The scale bar denotes the number of nucleotide differences per site. 


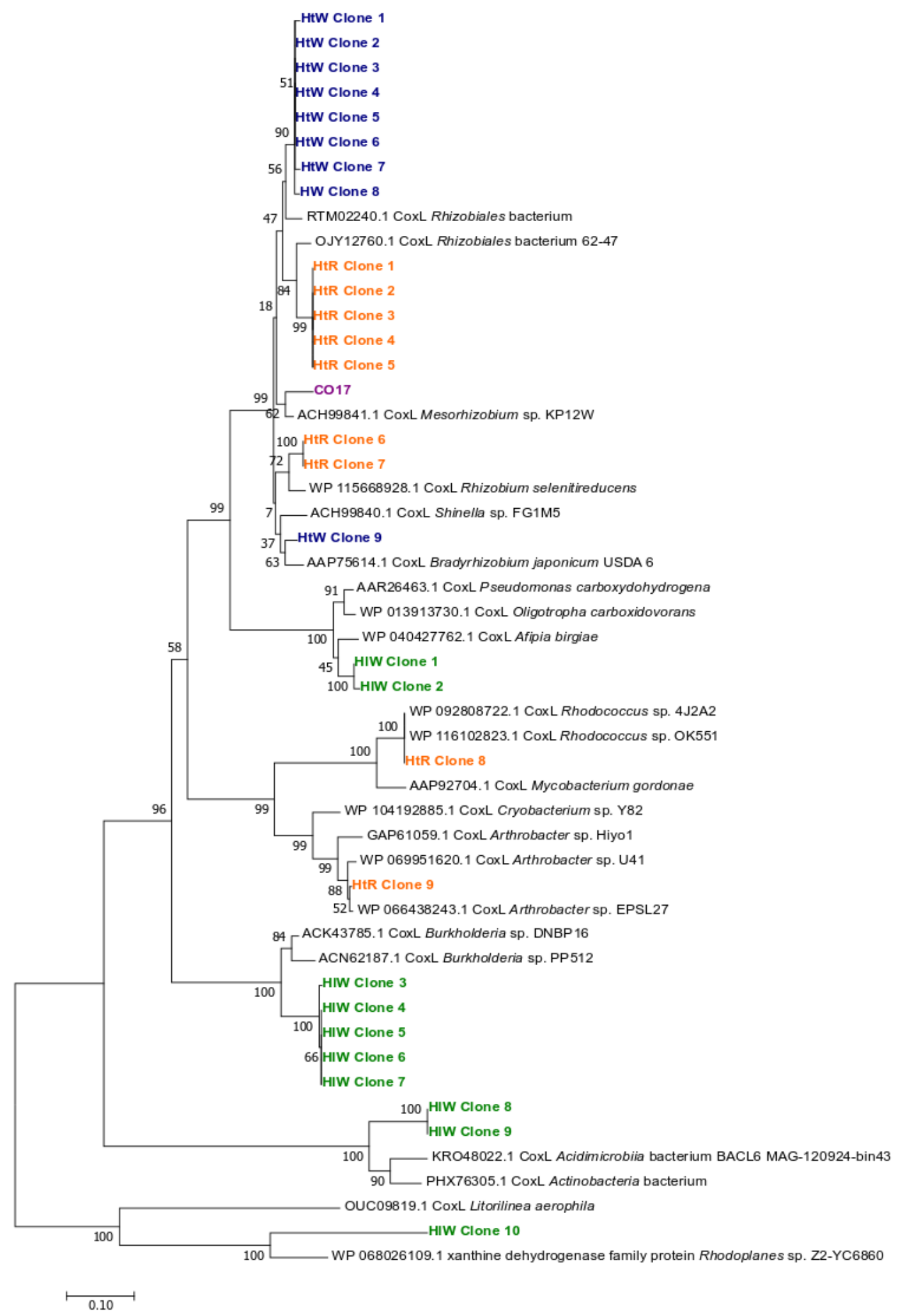

Figure 4: Phylogenetic tree of coxL PCR product sequences from CO-enrichment culture clones. Phylogeny of 1030 sequences and BLAST hits were inferred using the Neighbor-Joining method [1] of amino acid sequences in MEGA7 [2]. The percentage of replicate trees in which the associated taxa clustered together in the bootstrap [3] test (500 replicates) are shown next to the branches. Accession numbers are indicated before sequence names. $\mathrm{HtW}=$ woodland hawthorn samples (blue), $\mathrm{HtR}=$ roadside hawthorn samples (orange), $\mathrm{HIW}=$ woodland holly samples (green). For comparison, the CoxL amino acid sequence from isolate CO17 (purple) was also included. The scale bar denotes the number of amino acid differences per site. 


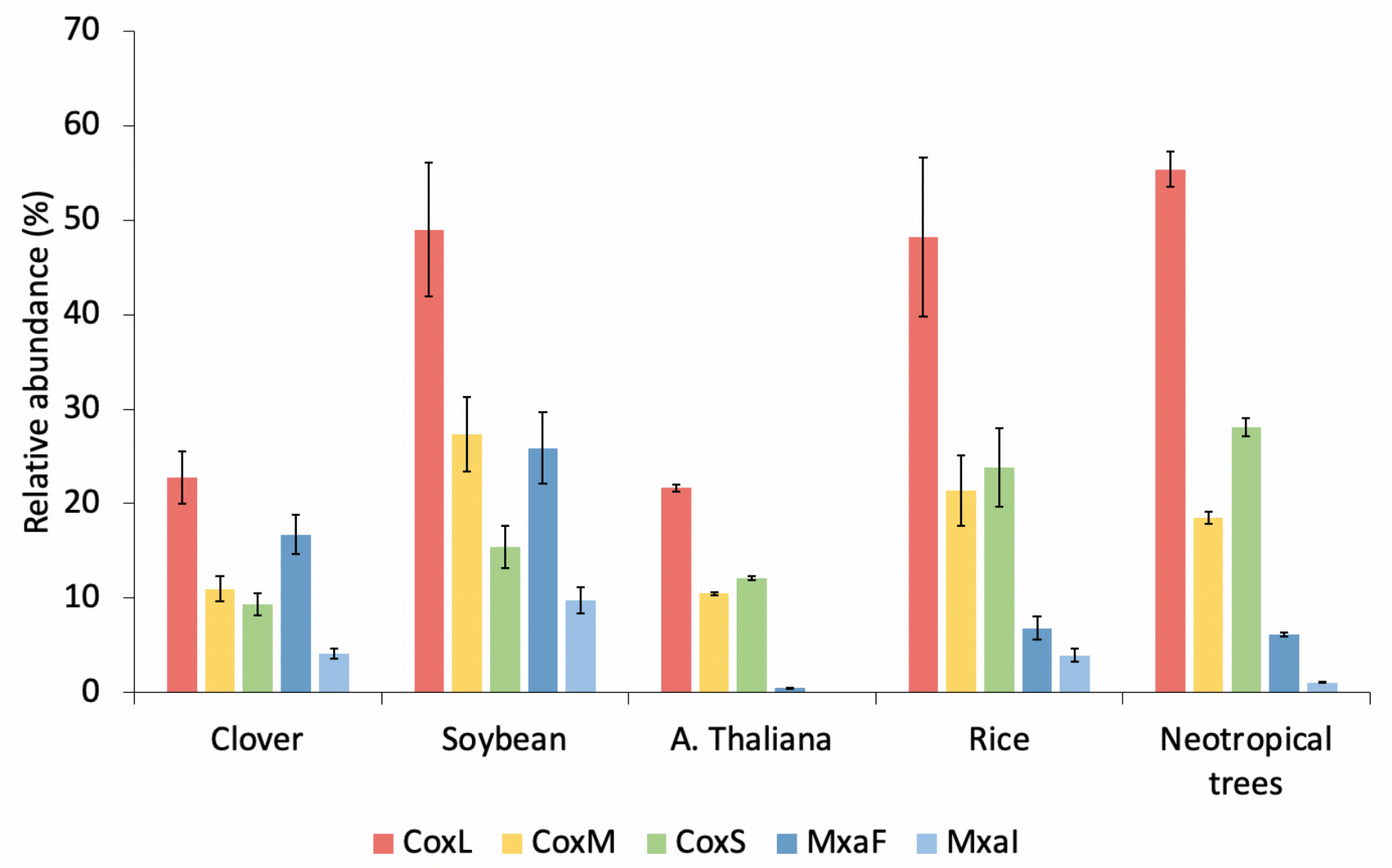

Figure 5: Abundance of cox gene homologues in phyllosphere metagenomes available on MG-RAST. Abundance of the large (coxL, red), medium (coxM, yellow) and small (coxS, green) subunits of CODH are shown in comparison with methanol dehydrogenase subunits ( $m x a F$, dark blue and $m x a l$, light blue), a common phyllosphere function. All functional genes were normalised using three single-copy housekeeping genes: RecA, Rho and RpoA. 
A)

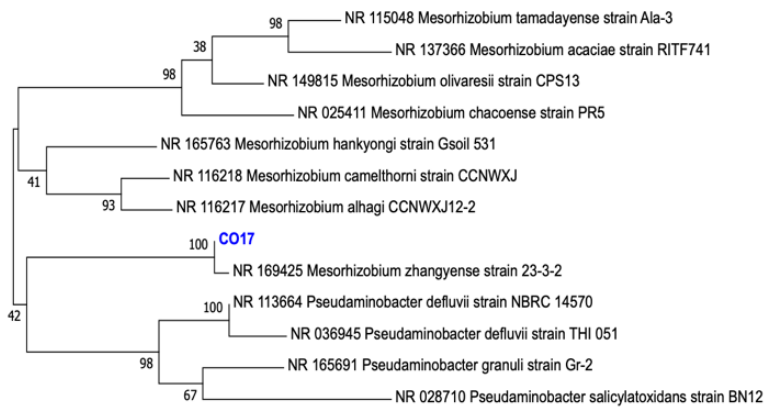

B)

Form I
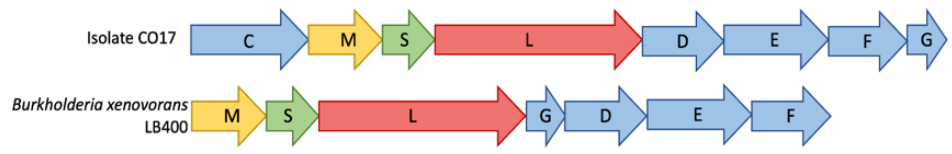

Form II

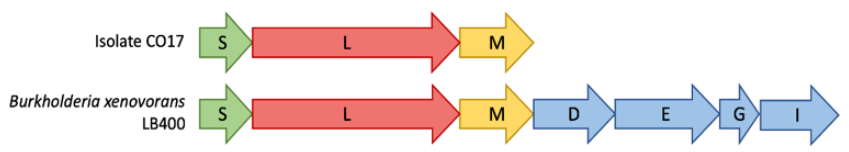

1044

1045

Figure 6: Characterisation of CO-oxidising isolate C017. A) 16S rRNA phylogeny of isolate CO17. Phylogeny of isolate and BLAST hits was inferred using the Neighbor-Joining method [1] of nucleotide sequences in MEGA7 [2]. The percentage of replicate trees in which the associated taxa clustered together in the bootstrap [3] test (500 replicates) are shown next to the branches. Accession numbers are indicated before genus names. The scale bar denotes the number of nucleotide differences per site. B) Arrangement of form I and form II cox genes in isolate CO17 and Burkholderia xenovorans LB400. The genes encoding the small (coxS), medium (coxM) and large (coxL) subunits are indicated in green, yellow and red, respectively. Accessory genes are indicated in blue. 